\title{
A Proposed Format for Mandibular Displacement Analysis in Fixed Prosthodontics
}

Timothy O. Hart

Loyola University Chicago

Follow this and additional works at: https://ecommons.luc.edu/luc_theses

Part of the Prosthodontics and Prosthodontology Commons

\section{Recommended Citation}

Hart, Timothy O., "A Proposed Format for Mandibular Displacement Analysis in Fixed Prosthodontics" (1983). Master's Theses. 3383.

https://ecommons.luc.edu/luc_theses/3383

This Thesis is brought to you for free and open access by the Theses and Dissertations at Loyola eCommons. It has been accepted for inclusion in Master's Theses by an authorized administrator of Loyola eCommons. For more information, please contact ecommons@luc.edu. (c) (i) $\Theta \Theta$

This work is licensed under a Creative Commons Attribution-Noncommercial-No Derivative Works 3.0 License. Copyright @ 1983 Timothy O. Hart 


\section{A PROPOSED FORMAT FOR MANDIBULAR \\ DISPLACEMENT ANALYSIS IN FIXED \\ PROSTHODONTICS}

BY
TIMOTHY 0. LHART D.D.S.

A Thesis Submitted to the Faculty of the Graduate School Of Loyola University of Chicago in Partial Fulfillment of the Requirements for the Degree of Master of Science

August

1983

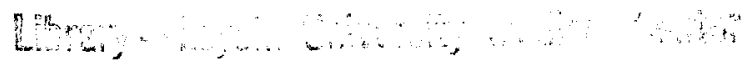




\section{ACKNOWLEDGEMENTS}

The author would like to express his appreciation to Dr. William Malone for the motivation and guidance he provided during the execution of this project. Dr. Malone's dedication to his students and his skill as a prosthodontic educator should serve as an example and a goal to others in his field.

The author is also indebted to Dr. James Sandrik and Dr. Douglas Bowman for their expert input and guidance. The participation of these men as members of the thesis committee proved indespensible in developing and carrying out this experiment. The expert assistance of Mr. Telespore Janovicz in the construction of the measuring device was essential to the success of this project and is deeply appreciated.

Finally, the author would like to express his deepest appreciation to his wife, Dr. Yvonne Balthazar, for both the help she provided with her prosthodontic expertise and her extraordinary patience and motivation. 
The author, Dr. Timothy Otto Hart, is the son of Hugh James Hart and Annette (Kahle) Hart. He was born February 16, 1952 in Waukegan, Illinois.

His elementary and secondary education were obtained in the public schools of Waukegan, Illinois.

In August, 1970 he entered Carthage College, Kenosha Wisconsin, and in June, 1975, he received the degree of Bachelor of Arts with a major in Biology. In August, 1973, he entered Loyola University School of Dentistry, and in June, 1977 he received the degree of Doctor of Dental Surgery.

In September, 1978, he entered the Prosthodontic Specialty and Masters in Oral Biology programs at Loyola University School of Dentistry. The Prosthodontic Specialty Certificate was awarded in May of 1980 .

In February of 1981, the author was awarded the Stanley Tylman Award, the national research award of the American Academy of Crown and Bridge Prosthodontics, for the research accomplishments described in this thesis. 
TABLE OF CONTENTS

Page

ACKNOWLEDGEMENTS ........................ i

VITA ...............................

LIST OF TABLES .................... . . . . v

LIST OF ILLUSTRATIONS . . . . . . . . . . . . . . . vi vi

Chapters

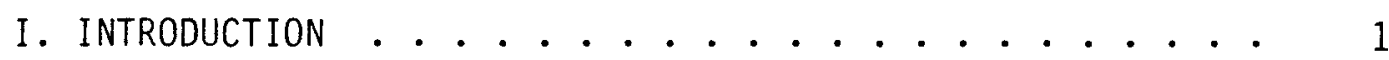

II. REVIEW OF THE LITERATURE . . . . . . . . . . 3

III. MATERIALS AND METHODS

Part I. Theoretical Considerations

and Instrument Design Specifications . . . . . 34

Part 2. Experimental Procedures ......... 62

IV. EXPERIMENTAL RESULTS . . . . . . . . . . . 65

V. DISCUSSION ............................ 71

VI. CONCLUSION . . . . . . . . . . . . . . 94

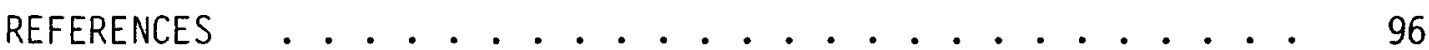




\section{LIST OF TABLES}

Tab le

Page

I. Means and Standard Variations of the Six

Parameters of Mandibular Displacement

66

II. Significant Temporal Comparisons Between

Experimental Runs of the Same Samples . . . . . . . 68

III. Means and Standard Deviations of the Vector Analysis Parameters of Mandibular Displacement ................... . . 69

IV. The Generalized Rotation Transform as adapted for Specific Use In This Investigation 


\section{LIST OF ILLUSTRATIONS}

Figure

1. Diagrammatic Demonstration of Pitch, Yaw and Roll as Applied to the Maxillary Assembly... . . . . . . . . . . 37

2. Modified Maxillary Member of Articulator . . . . . . . 41

3. Posterior View of Maxillary Assembly . . . . . . . . 42

4. Superior View of Maxillary Assembiy . . . . . . . . 43

5. Right Side View of Maxillary Assembly With Right Mirror Removal . . . . . . . . . . . . 44

6. Right Side View of Maxillary Assembly With Right Mirror in Place . . . . . . . . . . 45

7. Anterior View of Maxillary Assembly . . . . . . . . 47

8. Base Assembly .. . . . . . . . . . . . . . . 48

9. Superior View of Mounting Table . . . . . . . . . . 50

10. Lateral View of Mounting Table . . . . . . . . . 51

11. Anterior View of Mounting Table . . . . . . . . . 52

12. Photograph of Experimental Set-up . . . . . . . . . 54

13. Diagrammatic View of Apparatus from Superior Aspect . . . 57

14. Diagrammatic View of Apparatus from Anterior Aspect . . . 58

15. Diagrammatic Depiction of Optical Path (Lateral view) . . 60

16. Diagrammatic Depiction of Optical Path (Superior view) .............................. 61

17. Illustration of the Buhnergraph . . . . . . . . . 80

18. Schematic of Proposed System for More Accurate Angular Measurement . . . . . . . . . . . . 93 
INTRODUCTION

The relationship between the mandible and the maxilla is of paramount concern to the practice of dentistry. The importance of visualizing, measuring, and duplicating this relationship is reflected by the myriad of investigations found in the literature of the dental profession. The position and displacement of the mandibular teeth has been the concern of numerous types of studies. Such investigations involve various types of methods of measurement and, unfortunately, several different formats of reporting their results. No consistent format for the description of mandibular position is evident in the literature. This situation renders the meaningful comparison of results from different experiments impossible.

This investigation seeks to establish a meaningful format describing positional changes during mandibular displacement. When the location of the mandibular member of an articulator changes in reference to the maxillary portion, the total positional alteration can be described by the parameters of anterior-posterior displacement, left-right displacement, superior-inferior displacement, tilt in the coronal plane, tilt in the horizontal plane, and tilt in the saggital plane. Experimental description of such parameters of displacement can be accomplished by assigning each member of the articulator its own coordinate reference system. This study proposes 
a format of mandibular displacement description based on such a concept. An instrument which measures articulator displacement consistent with the derived format was designed and built. A simple experiment seeking to determine the influence of fabric screen matrices on the accuracy and stability of polyether registration materials was executed using the apparatus. Finally, a second format of spatial analysis based on vector analysis was derived in order to apply the principles of transformation matrices and displacement modeling to the data. This format was both interchangeable with the multi-coordinate system and conducive to analysis of local ramifications of articular displacement. 
CHAPTER II

\section{REVIEW OF THE LITERATURE}

\section{Introduction.}

The major purpose of this investigation is to establish a method of evaluating materials, techniques, and procedures that involve relating the mandibular arch to the maxillary arch. These procedures effect the relationship between the maxillary and mandibular members of an articulator. Prior to mounting casts, the two halves of the articulator are mechanically related to one another by means of the two simulated condylar areas and the incisal pin and table assembly. The clinical situation often arises in which a recording of maxillo-mandibular relationships is desired without the influence of the dentition. In such cases the two members of the articulator are related by means of the two condylar TMJ analogs alone. The transverse axis of an articulator is then described by the condylar positions. Procedures and materials that involve the inter-cast relationship have a direct effect on the position of this axis. It follows, therefore, that any evaluation of interocclusal registration materials and techniques involves the full description of any displacements of the articulator transverse axis resulting from such procedures. The achievement of this goal necessitated review of several concepts described in the dental and bio-mechanical literature. The pertinent concepts to review for this investigation would include the following: 
1. Biomechanical and kinematic considerations of joint positional analysis.

2. Basic axial theories of the temporomandibular joint and their applications to modeling mandibular position on an articulator.

3. Motion studies involving three-dimensional analysis of mandibular displacement.

4. Static maxillo-mandibular studies evaluating various interocclusal recording materials and techniques.

Biomechanical and Kinematic Considerations.

The problem of standardizing a descriptive format for defining mandibular position is unique to the field of dentistry. The review of the dental literature reveals no common, comprehensive, method of describing the position of the mandible in space. The development of descriptive systems for the positioning of an arbitrary body or solid in real or modeled space is, however, a goal common to several diverse fields of endeavor. Such disciplines as biomechanics, robotics, kinesiology (scientific), aeronautics, and computer graphics are all concerned with the three-dimensional description of bodies in space. A discussion of the several available methods of correct positional analysis is necessary to explain the justification for development of a specific format for three-dimensional mandibular description. The proposal for design of an apparatus for the evaluation of interocclusal records is subsequently based on this format. Therefore, design considerations must be derived as applications, modifications, or rejections of existing descriptive systems found in both the dental and non-dental literature. 
The nature of three-dimensional space.

Three-dimensional space can be conceptualized by two basic reference systems (Nielson and Vanlonkhuyzen, 1954). These two systems allow the location and attitude description of a body in space by means of either a rectangular or polar coordinate system.

The rectangular, or Cartesian, coordinate grid allows for the location of any given point in space by means of a single coordinate triplet. Each coordinate represents the projection of a point on the appropriate $(x, y$, or $z$ ) axis. Therefore, the format for a point would be $P(x, y, z)$. A line, or displacement, in the rectangular system is represented by two points, and a plane is defined by three points.

The polar coordinate system bases description of a point's position by means of angular displacements from a reference position, along with the radial distance of the point from the origin. This is best visualized by imagining a one-dimensional satellite. The craft's position in space, in relation to the earth, may be defined by the following parameters: (1) its radial distance from the earth's center (altitude), (2) its easterly progress in reference to the Greenwich Meridian (angular rotation), and (3) the angle formed by its altitude radius and the projection of that radius on the plane of the equator (declination). Therefore, a constant altitude displacement is represented by a pure angular displacement and a general displacement is represented by an angular displacement in addition to an altitude change. Depiction of a plane involves at least three angular displacements and usually two or more changes in radius. Although such a description can be accomplished easily by means of transformation 
matrices, it is obvious that the polar coordinate system is rather inconvenient to utilize when conceptualizing anything more than a point's position. Likewise, the rectangular system is very nondescriptive of angular displacements.

Descriptions of objects in space.

When an object in space displaces from a start position to an ending position, the locations of most or all of the points on that object changes. Description of such a displacement could be accomplished by accounting for the movement of three or more of the points on the object in either Cartesian or polar coordinate systems. Such a methodology would be computationally complex. Additionally, the displacement parameters generated would be nearly useless for human conceptualization. Both these complexities can be simplified by means of multiple, or global-local, coordinate systems.

Each of the previously mentioned coordinate formats have one point in common: the origin. The origin is the arbitrary center reference point that serves as location $P(0,0,0)$ in the Cartesian system and the vertex of all angles in the polar system. It is important to realize that the origin is an arbitrarily chosen point. Also recall that the simplest positional description in each system involves a single point. Therefore, if we could reduce the complexity of positional description into analysis of point displacements conceptualization would be greatly enhanced. An object is, however, an infinite collection of points. During a displacement, all the points that are associated with and actually belonging to 
the object move in a complex manner relative to the origin of the reference system. The complexity of these point movements could be greatly reduced if their displacement were related to an arbitrary single point that bears a constant relationship with each point of the object (Hungerford, 1978). Such a simplification is possible by assigning a secondary, or local, reference system to the object. Thus the displacement of an object can be described in a two-step manner: (1) displacement of the local reference system's origin in relation to the overall, or global, coordinate system, and (2) positional changes of the points belonging to the object in relation to its own, local, coordinate system. Note that both global and local reference systems need not be of the same format. In that all points of the body's local reference system maintain a constant distance, or radius, from the local origin, the polar coordinate system would allow for a more ideal descriptive format for attitudinal change. Furthermore, if one of the three axes of the local polar system were chosen as an axis of orientation, attitude change of the object could be described by the familiar aeronautical terms of pitch, yaw, and roll. Secondly, in that movement of a point in space is best visualized by $x, y$, and $z$ displacement, the movement of the entire object can be described by the displacement of its local origin in a global rectangular coordinate system. These mathematical considerations can be applied to the adaptation of a system for describing mandibular displacements. Before discussion of a fully descriptive system, the historical background of measurement of motion between two bodies in space from a 
biological siandpoint will be considered.

Kinematic considerations pertaining to the measurement of motion between two body segments.

Historically, consideration of vertebrate joint dynamics has emphasized modeling of the joint, itself, as opposed to segmental analysis of its participating members (Kinzel, 1972). Such an approach has led to a progression of joint models beginning with the simple revolute model (one degree of freedom) and leading to complex, fully descriptive (six degrees of freedom) joint models (Kinze1, 1972; Orin, et a1, 1979; Lewis and Lew, 1972; Lewis and Lew, 1978; Roedema, et ar, 1978).

The simplest joint model is the revolute joint. It permits rotation about a single axis and involves one degree of freedom. Only one rotation angle needs to be measured to describe its motion. The best example of the revolute joint in dentistry is, of course, the "barn-door" hinge articulator. A planar joint is an articulation that involves one joint member gliding, or translating, in respect to the other. A planar joint permits from one to three degrees of freedom. Translation along a straight surface would involve one degree of freedom while a curvilinear glide would entail two degrees of freedom. A third degree of freedom would be manifested by a concurrent rotation of one joint member in relation to the other. Such a model has been used for knee joint description (Freudenstein and Woo, 1969) and would be descriptive of current condyle-oriented articulators (Guichet, 1977). The classic spherical joint model (Eberhart et al, 1947) encompasses three (a11 rotational) degrees of freedom 
and is most often applied to the hip joint. It involves all the motions typical of an object's attitudinal change in space utilizing the polar parameters of pitch, yaw, and roll of a local orientation axis.

The advent of anthropomorphic mechanisms for industrial and research applications has necessitated the development of actuator systems capable of mimicking joint movements in all possible degrees of freedom (Stepanenko and Vukobratovic, 1976). Such movements can be produced through the use of various configurations. A relatively nonanatomical but computationaliy efficient method involves the sequential actuation of six or more links oriented in such a fashion as to position any given link $90^{\circ}$ to any other link (Orin, et al, 1979). Such a system has been used in dentistry to duplicate mandibular movement (Abler, 1972) and to evaluate similarities in articulator motions (Beck and Knap, 1976). Other actuator systems involve manipulation of joint member axes in a more anatomical fashion. An industrial manipulator can be constructed in a fashion similar to the human arm and subsequently its motion produced by the six parameters of $x, y$, and $z$ displacement; and rol1, pitch, and yaw (Snyder, 1980). A11 these motions are produced by combinations of revolute and bi-revolute (universal) joints. Just as actuators can be employed to produce joint-like motions in mechanical devices, revolute sensing devices can be utilized to detect the motions of actual anatomical joints in experimental situations (Kinzel, 1972). The output of such devices as potentiometers and radial optical sensors (Abler, 1972) can be integrated through data processing to produce a model of joint motion 
(Lewis and Lew, 1977). Kinzel (1972) used kinematic linkages of six potentiometers and seven segments to create a model of the shoulder of a dog. Rather than the previously described global-local coordinate system, Kinzel defined a unique screw axis for any given joint displacement.

Regardless of how the actual motion takes place, the displacement of the moving body from position 1 to position 2 can always be represented as a rotation about, and a translation along, a unique axis located somewhere in the fixed body. This axis is directly analogous to the instant center of rotation for plane motion and is called the screw axis.

This particular spatial descriptive system has specific application to relating joint motion to articular surface morphology, and consequently will not be discussed in further detail.

Roedema, et al (1978) utilized a computer program to model the movements of a theoretical mandible based on the Denar D5A articulator. A global-local type of system was employed in this simulation. A rectangular global system of reference was used while the local system consisted of polar coordinates originating in the left condyle. The main purpose of Roedema's model was to relate mandibular movements to occlusal morphology. No attempt was made to relate such a system to clinical situations.

It can be concluded that numerous authors have dealt with the problems of anatomical joint description. Joint models depicting less than the full six degrees of freedom tend to emphasize mimicking of the artriculation, itself, and include the following:

1 Degree of Freedom

-the revolute joint

-The straight planar joint 
2 Degree of Freedom

3 Degree of Freedom -curvilinear planar joint

-non-rotating spherical joint

-curvilinear planar joint with rotation

-full spherical joint

Joint modeling systems that involve six degrees of freedom tend to emphasize the relative motion of one member in reference to the other. Such models may involve measuring and duplication instruments that do not necessarily bear any resemblance to the morphology of the joint in question. This is because an articulation involves a motion between two solids limited by the joint and its associated musculature; rather than a joint actually moving its members by means of strict mechanical parameters. Therefore, the important concept to keep in mind when evaluating six-degree-of-freedom models of joint motion is that such models describe the motion between two body parts as they are constrained by the joint, rather than directly attempting to model the joint, itself.

Basic Axial Theories of the Temporomandibular Joint and Their Applications to Modeling Mandibular Position on an Articulator.

The dental literature is repleat with articles extolling the various theories, descriptions, and concepts relating to the axes of the human mandible and its analog, the articulator. The horizontal axis of the intercondylar area has received the most scrutiny of all such potential axes. The ultimate goal envisioned with the location and description of a repeatable horizontal axis is the initiation of proper transfer of a patient's casts to an adjustable articulator 
(Granger, 1952). It is important to keep in mind, however, that the act of defining or locating a repeatable horizontal axis need not have any basis in human morphology. Its only purpose is to act as a foundation of orientation for the sake of determining parameters for modeling mandibular motion mathematically (Roedema, 1978) or mechanically (Granger, 1952).

The transverse axis of the mandible has been attributed to numerous definitions and synonyms. Such terms as hinge axis, kinematic axis, arbitrary axis, horizontal axis, split axis, centric relating axis, capitular and tubercular axis, instantaneous axis, and intercondylar axis are found throughout the literature. The definition for any one of these axes and its accompanying application can be based, singularly or in combination, on anatomical, functional, or modeling considerations. Aull (1963) summarized the historical discussion of transverse axis into four schools of thought. These schools of thought represented functional, morphological, and application definitions of several axes. A more complete list of axial definitions and implications will be considered as follows:

From Aull's schools of thought:

1. Absolute hinge axis

2. Arbitrary hinge axis

3. Non-existence of transverse axis

4. Split-axis rotation ("transographics")

Additional schools of thought found in the literature:

5. Combination Capitular and Tubercular axes

6. Instantaneous axis

7. Kinematic axis 
Absolute hinge axis (syn.: intercondylar axis, anatomical axis, centric relation axis).

The original and classic gnathologists supported the existence of an absolute hinge axis (Lucia, 1960; McCollum and Stuart, 1965). Such an axis exists on an anatomical basis in that it coincides with the horizontal axes of rotation of both condyles. Also, each of the condyles possesses an anatomically defined vertical axis which must be located in order to evaluate lateral movements of the teeth. The mandible, therefore, can undergo a limited amount of pure nontranslatory rotation about the horizontal axis. This limited rotary movement can be used to find the specific location of the absolute hinge axis by means of a hinge axis locator (McCollum and Stuart, 1965), and subsequently transferred to an articulator. The articulator's adjustable condylar elements are coincident with the anatomically defined and dynamically located hinge axis. The major constraints that delineate the absolute axis philosophy in respect to the more "liberal" gnathological concepts are (1) the insistence of the existence of an anatomical definition (e.g. rearmost, uppermost, midmost, position of the condyle) of centric relation, and (2) the opinion that since the condyles each have a vertical axis of rotation, a fully adjustable articulator must have an adjustable intercondylar distance and therefore "in order to be a relational likeness of jaw movement and positions, the instrument must also be a mechanical likeness" (Stuart, 1979). It will be later demonstrated that the gnathologists' contention of strict anatomcial replication of temporomandibular articulation did indeed result in a legitimate modeling of the maxillo- 
mandibular relationship. However, these self-imposed mechanical constraints have possibly mislead thousands of practicing dentists into utilization of a correct, but greatly overcomplicated, means of mandibular modeling.

Arbitrary hinge axis (syn.: facebow axis, earbow axis).

The major principle defended by those ascribing to the concept of arbitrary hinge axis states that the location of a true hinge axis does, indeed, exist, but is only of academic interest (Craddock, 1952). The arbitrary hinge axis is based on a population average and is referenced to external anatomical landmarks. It is further believed that such a position represents an axis that is sufficiently close to the real axis to be utilized for all articulator mountings. Recent investigations (Walker, 1980) have disputed the viability of such an arbitrary axis. In addition to determining that an arbitrary hinge axis location for the total population does not exist, Walker concluded that

(1) the wide dispersion of true anatomic axis point locations indicates that any measurement chosen for an arbitrary axis will create a large error and poor accuracy, and (2) very few individuals have the same true axis point locations on both sides of the face.

Non-existence of hinge axis.

Beck (1959) concluded that the so-called rotation of the condyles actually represents the integration of numerous translations and rotations. Since the opening and closing hinge movement of the mandible, together with its fragmentary movements, cannot be repeated 
by the opening and closing movements of an articulator, which is about one axis only, the concept of a hinge axis is invalid. In other words, the non-existent hinge axis viewpoint recognizes that a locatable transverse axis does not exist within the constraints of anatomical definition. Furthermore, the lack of anatomical definition precludes the possible formation of a mechanical model of jaw movement.

Split axis (syn.: transographics).

The transographics school of thought also bases its conclusions solely on anatomical observations and derives its mechanical modeling only from theoretical considerations. It is recognized that the terminal hinge position usually manifests itself asymetrically on the patient's head (Aul1, 1963). This anatomic condylar asymmetry, in addition to the frequent observation of off-vertical mandibular opening, is the basis for the assumed existence of two independent hinge axes (one in each condyle). The validity of transographics is highly questionable. The transographic constraints of mandibular motion would involve the simultaneous rotation of a rigid body about two static axes. One need only to attempt visualization of such a situation in order to evaluate the theory of transographics.

Combination Capitular and Tubercular axes.

Another concept of axes locations involves the existence of two bilateral rotational centers which determine mandibular motion (Moss, 1975). According to this philosophy, at no time, under any normal conditions, will an isolated hinge rotation of the condyle 
occur in the lower joint compartment without a simultaneous rotation in the upper joint compartment about the tubercular axis (Nevakeri, 1960). Such a theory is based on the true mechanical axiom that states "in any mechanical situation where rotation occurs simultaneously about two or more axes of movement, one can always describe a resultant axis rotation." Moss concludes that

it has been repeatedly demonstrated by many workers independent of each other [unfortunately, Dr. Moss does not volunteer citations of, or references to, any of the 'many workers'] that what appears to be a 'translation' of the condylar head during protrusive and retrusive positioning of the mandible, for example, is, in fact, a motion of rotation at the upper joint compartment with an axis of movement passing transversely through a given articular tubercle.

This point is subsequently "proven" by a series of drawings that illustrate the true center of mandibular rotation (near the mandibular foramen) as it is derived from simultaneous rotations about tubercular and capitular axes. One must realize, however, that when such a "true center of mandibular motion" is described along with denial of any translatory displacement, the phenomonom of instantaneous axis of rotation is manifested. The instantaneous axis of rotation is another legitimate means of describing a body in space, but, as will be discussed below, lends itself poorly to mechanical modeling. Unfortunately, Moss fails to recognize that non-consideration of translation results in a dynamic center of rotation whose location is only temporarily at the mandibular foramen. Furthermore, no experimental studies, mechanical models, or mathematical models are cited or proposed for the substantiation of the combination capitular 
and tubercular axes theory.

Instantaneous axis.

Calculation of muscle moments, moment arms, and forces requires the ability to locate the center of rotation of a body at given instants in time. Therefore, when such variables are under consideration, the dynamically changing axis, or instantaneous center of rotation, must be taken into consideration (Grant, 1973). The instantaneous axis is simply the center of rotation defined by the total movement of a body in space at a given coordinate in time. The important distinction involved with the concept of instantaneous center of rotation (ICR) is that definition of the axis' location on the body and its position in the space-time coordinate system assumes that all aspects of the body's motion are attributed to rotation. This constraint is necessary in order to facilitate the evaluation of static and dynamic equilibria involved in muscular work. The use of ICR has created controversy as to the validity of hinge axis theories (Grant, 1974; Stern, 1974). Authors supporting ICR have shown that theoretical calculations involving mandibulo-cranial force distributions yield far more realistic results using a dynamic axis rather than an intercondylar axis (Grant, 1973). Those supporting an intercondylar axis insist that it is equally valid to take moments about the condyle when analyzing mandibular statics (Stern, 1974).

The disagreement involving these two viewpoints can be easily resolved when one recalls that proper positional and displacement analysis and modeling of a body in space can be executed under various 
modalities. The selection of which modeling system to use is influenced by both the desired degree of anatomical and dynamic complexity of the analysis and the intended goal to be accomplished by the resulting model. Therefore, it can be concluded that both ICR and intercondylar axis analysis are acceptable models for mandibular movement. ICR modeling allows for calculations of various muscle force variables, while an intercondylar axis format lends itself to simpler mechanical modeling of motion displacements involved with the TMJ articulation.

Kinematic axis.

The kinematic axis is often considered synonomous with the original absolute axis of McCollum and Stuart (1965). It is viewed as that axis about which the mandible (with the condyles in centric relation) executes pure rotation in the first $10^{\circ}$ to $15^{\circ}$ of angular displacement. The kinematic axis is determined clinically by use of a hinge axis locator. This device is simply a U-shaped apparatus that, through rigid attachment to the mandibular teeth, becomes, by mechanical definition, part of the mandible. The cross-bar of the "U" is located anterior to the incision, while the "tails" of the "U" possess pointers that are, on the so-called $90^{\circ}$ systems (lucia, 1960), parallel to the anterior crossbar. The pointers are adjustable in two dimensions and represent, ideally, coincidence with the actual rotational axis of the mandible. The major distinction between absolute hinge axis and kinematic axis is as follows: in that the kinematic axis determination is made through actual clinical obser- 
vation, it need not represent an axis defined by anatomical considerations. According to Preston (1979)

Condyles are asymmetric bodies confined only to certain limits of questionable exactness by muscles and ligaments. The condyles are asymmetrically positioned in their relation to the cranium and to one another. The mandible functions as a unit in spite of these asymmetries, and the resulting movement may be or resemble [a point or] an arc. The operator seeking an axis either can or cannot locate the arc center of the clutch.

Further thought into the nature of the kinematic axis locator reveals that is pointers do not represent coincidence with the mandible's axis (Gregory, et al, 1969). Instead, only the tips of the pointers represent two points (not lines) that exist on the hinge axis. Therefore,

the axis locator is not an axle, it is a seeker of a center of motion. It does not matter if the points located are connected to the side arms by a right angle, any other angle, or a complex spiral, as long as that connection is rigid. Only the point of termination (the tip of the stylus) has validity, and it only has validity in relation to the overall apparatus and its movement. (Preston, 1979)

The ultimate purpose of kinematic hinge axis location is to create a clinical situation that is directly equivalent to the mechanical modeling constraints of the articulator. Location of the kinematic axis, when achievable, is specifically for the purpose of transferring an arc of rotation in the saggital plane from the patient to an articulator. The articulator, by means of mechanical design, possesses a morphologically determined transverse axis in the intercondylar vicinity. If an analogous kinematic axis can be determined 
in the patient, the first major step to proper modeling is accomplished; positioning of the clinically observed area of dental interdigitation in an equivalent spatial relationship with the articulator transverse axis (not the articulator's "condyles"). Both members of an acron articulator possess mechanical representations of the transverse axis. The axis is depicted by an imaginary line traversing through the condylar housings of the maxillary member. In the transferred centric relating position, the intercondylar axis of the mandibular member is, ideally, positioned identical in space to the maxi11 ary member's transverse axis. The relationship of the patient's maxillary arch to the articulator is accomplished by creating a coincidence of the clinical kinematic axis with the articulator maxillary member's transverse axis. The subsequent attempted coincidence of the mandibular member's transverse axis with that of the maxillary member is accomplished by means of the interocclusal record procedure. Any error in interocclusal registration techniques or materials will, therefore, result in a non-coincidence of the articulator maxillary transverse axis and the articulator mandibular transverse axis. Logically, evaluation of the accuracy of various techniques and materials should involve a full three-dimensional analysis of any such non-coincidence brought about by descrepancies in interocclusal registration procedures.

In conclusion, the kinematic axis, when achievable, is the most ideal clinically determined axis to be utilized for mechanical modeling. (Preston, 1979). Furthermore, an ideal acron articulator mounting is created when the following three axes coincide: 
1. The kinematic axis as determined by a hinge axis 10cator.

2. The articulator maxillary member transverse axis as determined by facebow transfer based on kinematic axis location.

3. The articulator mandibular member transverse (or inter"condylar") axis as determined by interocclusal record procedures.

Motion Studies Involving Three-dimensional Analysis of Mandibular Displacement.

With the possible exception of the femoral-tibial articulation, the temporo-mandibular system has been the subject of more intensive and varied attempts of positional description than any other joint in the body. A review of the literature reveals that attempts of describing mandibular motion or position usually involve either point motion studies or descriptions of single or multiple axial motions. Numerous point motion studies have been undertaken. Posselt (1952) and others (Knap, 1973) described the envelope of mandibular vertical motion as depicted by a tracing point related to the mandibular incisor utilizing the skull as the reference body. Brash, et al (1956) used lightweight hollow tubular clutches which housed light sources. The tubes were affixed to clutches and were photographed using mirrors and a stereoscopic movie camera. More recent investigations have attempted to decrease the mass of any intraoral apparatus to be tracked, thereby diminishing interference to normal mandibular movement. Such methods include photoelectric cell tracking (Waysenson and Salomon, 1977; Jemmt, 1979) of incision related points. 
Cementation of magnets to the teeth and subsequent electromagnetic tracking also resulted in a relatively non-interfering apparatus (Jankelson, 1979; Jankelson, 1975; Lewin et al, 1976). Perhaps the least interfering, and most exotic, method was utilized by Wasenson and Salomom (1979), in which a soft tissue mounted point source of radioactivity was positioned on the patient's chin and tracked with a gamma sensitive real-time camera.

The most important information derived from point motion studies involves detection of timing cycles (Jemmt, 1979). The method of anterior point tracking yields no meaningful parameters of condylar or mandibular position in space. To do so requires knowledge of the position of three points on the mandible at known instants and/or identification of all six parameters of positional change involving an identifiable axis of orientation.

Several studies have been undertaken in an attempt to accurately record and analyze mandibular motion in detail greater than single point motion. Of the numerous investigations attempting this goal, there are a number of studies that do deal directly or indirectly with six degrees of freedom. Abler (1972) designed and constructed a motion sensor/duplicator system which used a seven link, six potentiometer kinematic linkage system to measure and reproduce mandibular motion. The devices were based on the principles of the Newton-Euler open chain kinematics model as described by Orin, et al (1979). This is similar to the type of mathematical model that Kinzel (1972) util- 
ized to establish the motion parameters of the canine shoulder. The linkage apparatus in both cases were nonanatomic and located external to the joint members. Abler's system has since been utilized in various investigations. Knap, et al (1970) used the sensing mechanism of six potentiometers arranged in a precision linkage to record and graphically reproduce various envelopes of motion. Beck and Knap (1976) conducted a more thorough investigation of comparing human mandibular motion with the movement of various fully adjustable articulators. Abler's device was adapted to receive positional data from both the patient and the articulator. The duplicity of the mountings was evaluated by means of angular measurements of given envelope parameters. In that Abler's device was designed with a full six-degrees-of-freedom kinematic link chain, the device was quite applicable for this purpose. In fact, the investigators involved could have utilized the device to describe mandibular motion in a more meaningful, axis oriented, manner.

Messerman, et al (1967) devised a dynamic measuring system that involved six independent linear motion detectors on a kinematic facebow arrangement. The apparatus, based on the well known engineering principles of positional analysis (Rosenblatt, 1967), utilized an arbitrary and consistent axis of orientation located in the proximity of the middle of the path of the instantaneous center of mandibular closure. Furthermore, a dynamic replicator, the Case Gnathic Replicator, was constructed with servomotors acting on an orientation axis of the patient's casts identical to the axis defined by the linear photoreceptor arrays on the facebow. Such a system allowed Messerman 
to "play back" stored mandibular motions, along with providing an exhaustive database for additional analysis, such as determination of instant centers of rotation (Messerman, et al, 1969).

Lee (1969) developed a unique system that utilized high speed handpieces cutting into clear acrylic blocks. The apparatus framework was similar in design to a conventional earbow. The actual recording of mandibular motion occurred as three-dimensional tracings (grooves), in each of three blocks, for given mandibular border motions. The raw data, therefore, represented the rectangular displacement of the vertices of a triangle, thus defining the position of a plane possessing a constant relationship to the mandible. These data were greatly enhanced by aligning the reference position of two of the acrylic blocks with the kinematically determined hinge axis. Such a procedure established an arbitrary, functionally based, mandibular transverse axis that is coincident with the orientation axis of an articulator. Analysis of data (McCoy, 1976) produced in this manner resulted in a clinically usable model of mandibular motion and eventually led to the design of one of the two major fixed-condylar semi-adjustable articulators currently available (Lundeen, 1979).

The following is a summary of the three major systems, described in recent literature, that legitimately analyze mandibular movement in space (i.e. six degrees of freedom):

1. DEVICE: DESIGNER: MAJOR DESIGN FEATURE:
-7 segment kinematic linkage.

-Abler

- Utilizes open chain system of 6 kinematic linkages, each with a potentiometer, anterior to mandibular activity. 
3-D DESCRIPTIVE FOR-

MAT:

-Envelopes of motion as viewed in the $X Y, Y Z$, and $X Z$ planes.

2. DEVICE:

DESIGNER:

-Case Gnathic Replicator.

MAJOR DESIGN FEATURE: -6 linear opto-electronic transducers

3-D DESCRIPTIVE FOR-

MAT :

in a face-bow type of arrangement.

-Duplicates motion utilizing arbitrary but consistent axes of orientation located in the proximity of the ramus.

3. DEVICE :

DESIGNER:

- Lee Mandibular Movement Recorder.

-Lee

MAJOR DESIGN FEATURE: -Raw data produced by three-dimensional 3-D DESCRIPTIVE FORMAT :

tracings in acrylic blocks.

-Since two blocks oriented with clinically determined kinematic axis, motion is described utilizing the transverse condylar axis of the articulator as the axis of orientation.

Static Maxi110-mandibular Studies Evaluating Various Interocclusal Recording Materials and Techniques.

The methodologies of investigations into the accuracy of interocclusal record procedures and/or articulator mountings can be divided into three major categories: (1) qualitative evaluations of interocclusal records, (2) quantitative studies of interocclusal records, and (3) hinge axis location investigations. The emphasis of this review will deal with the techniques of geometrical analysis utilized by these studies, as opposed to their findings. Thus, experimental technique will be the principle criterion for the mentioning of any particular study.

Qualitative evaluations of interocclusal records. 
The object of a qualitative evaluation of articulator mounting or interocclusal registration is the confirmation of repeatability of a given procedure. The data derived from such investigations is boolean in nature and thereby is not subject to the requirements of true threedimensional displacement analysis. In other words, when the desired result is only a "fit or no fit" determination, the means of displacement evaluation need only fulfill one criterion: the evaluation procedure must produce a "no-go" or "no-fit" situation if displacement occurred. The majority of studies in this category involve the split cast technique (Needles, 1923; Lauritzen and Wolford, 1964; Lucia, 1964). This method entails specific notching and lubrication of the bases of the casts. An initial interocclusal record is used to mount the casts in the articulator. One of the casts is then separated from its mounting stone and a subsequent interocclusal record is interposed between the maxillary and mandibular arches. If the articulator member (usually the maxillary) and mounting stone assembly can close and interdigitate with the notches in the cast, a "fit" is assumed and the interocclusal record is verified. Schwarz and Gear (1975) further simplified the procedure by recommending the use of children's construction bricks to relate the cast to the mounting stone.

Other methods of mounting verification include the use of hinge axis oriented devices such as the Buhnergraph and Vericheck. Since these devices also have quantitative uses, they will be discussed below. Quantitative studies of interocclusal records.

Devices and measuring techniques designed to evaluate maxilo- 
mandibular relationships can be classified according to the following scheme:

1. Analysis of two-dimensional tracings and pointer positions.

2. Minor measurement of commercial articulator displacements (onedimensional).

3. Measurement of arbitrary cast landmark displacement.

Devices that create two-dimensional tracings that are representative of three-dimensional mandibular position are frequently encountered in the dental literature. Kabcenell (1964) developed a recording apparatus which consisted of a maxillary stylus and mandibular recording plate, each of which was attached at the midline to bands cemented to incisor teeth. A piece of paper was placed on the plate and closure was initiated. The resulting pin-prick created by the stylus was measured. The antero-posterior distance between $\mathrm{CO}$ and several types of $C R$ was analyzed. Apparentiy, no specific attempt to orient the apparatus consistently with any transverse axis was made. Therefore, it was impossible to determine how much true anterior-posterior displacement is indicated by the change in stylus position.

Kass and Knap (1974) also utilized a pin position type of apparatus to evaluate changes in maxi110-mandibular relationships occuring in displacement from initial retruded contact to the intercuspal position. A unique dower positioning device was fabricated which allowed consistent placement of L-shaped measuring dowels to the maxillary and mandibular casts. "The maxillary measuring component [dowel] was positioned relative to the maxillary cast and secured by 
a mounting bracket. This bracket enabled the measuring component to be repeatedly positioned at the same distance from [and consistent orientation to] the transverse axis of the articulator." The mandibular measuring component was related to the mandibular cast by means of a metal referencing block. The absolute distance between dowels, measured in a plane defined by the transverse axis and mounting bracket (which was essentially a $126 \mathrm{~mm}$ line of radius), was determined for each run of the experiment. The only potential drawback of the experiment involves lack of consideration of any possible medio-lateral component of the inter-dowel distance. In order to compare anterior-posterior relationships of the casts (in the context of the study), only the movement perpendicular to the transverse axis should have been determined. This shortcoming occurred only in the measuring procedures, however. The basic design of the apparatus would have allowed for such a determination.

Ingervall, et al (1971) and Helkimo, et al (1973) utilized an intraoral tracing procedure to compare interocclusal relationships (specifically various methods of CR determination). A graphical representation of mandibular position was created by means of a pin-and glass-slide device related to intraoral acrylic clutches. Both the anterior-posterior and medio-laterial components of any displacements created by $C R$ record procedures were measured with vernier calipers. The plane of reference for this system was defined by the maxillary clutch position. Grasso and Sharry (1968) developed an almost identical method of maxillo-mandibular evaluation. Intra-oral gothic arch 
clutches were used to record various mandibular positions. Minimization of human error involved in caliper manipulation was a major additional design consideration. Therefore, the anterior-posterior and medio-laterial components of mandibular position were measured utilizing a fixed precision record-accepting, two-dimensional caliper table. The system's plane of reference was also determined by maxillary clutch position.

Investigations utilizing minor measurement of commercial articulator displacements involve the measurement of displacement in one dimension and their simplicity is typified by two particular studies. Brewer (1963) positioned a dial indicator above a Hanau H2 and measured the horizontal displacement of the upper member of the articulator caused by various interocclusal records and plaster cast expansion. Parker, et al (1974) compared passive maxillo-mandibular records with functionally generated records. A dial guage was rigidly attached to the back of an articulator and adjusted to contact the posterior aspect of the maxillary cast. The anterior-posterior displacement of the cast after remount procedures was evaluated.

Several studies involve the measurement of arbitrary cast landmark displacement. Millstein, et al $(1971,1973,1975)$ devised a system for evaluating vertical dimension alterations caused by interocclusal registration materials. A device which applied pure vertical pressure, at $172 \mathrm{psi}$, to the maxillary cast was constructed. Three dots were placed at arbitrary points on the land area of the maxillary cast. The vertical displacement of these dots was determined by means 
of a measuring telescope. The positions of the dots were determined at maximum intercuspation, during application of pressure to the recording medium, and during replacement of the maxillary cast into the record. No attempt was made to relate these data to clinical or articulator hinge axis.

Strohaver (1972) evaluated maxillo-mandibular relationships by means of multiple extension rods imbedded in the casts of one individual. Five triangles were described by this system "which permitted measurements to be made both between the relationships of the two casts and the relationships of the mandibular cast to the axis of the articulator." These measurements were made by determining the distances between the rod ends with a vernier caliper. No attempt was made to offer descriptive data to evaluate the alteration of hinge axis position. The results merely consisted of sides and angles of the various triangles, all of which were "lumped" into one analysis of variance procedure. No provision was made for description of positional alteration of the plane described by each of the triangles.

Hinge axis location investigations.

The Buhnergraph is a device that was designed to provide verification capabilities without the necessity of using split cast technique (Long, 1970). The apparatus consists of a wide "U"-shaped piece of aluminum machined to mount on the mandibular member of a Whip-mix articulator. The Buhnergraph is mounted by replacing the condyles with the aluminum block. Two movable pointers are housed in the block lateral to the maxillary condylar housings. These pointers lie coin- 
cident with the articulator transverse axis. Duplication of an interocclusal record is verified in the following manner:

i. The maxillary cast is mounted using (a) an arbitrary facebow if $C 0$ records or equilevel CR records are to be verified, or (b) a kinematic axis facebow if "true" CR is to be verified using records of varying thickness.

ii. Two or more appropriate interocclusal recordings are made on the patient.

iii. The Buhnergraph is fastened to the mandibular member of the articulator and pieces of graph paper are affixed to the condylar housing.

iv. The first record is placed on the articulator and the "position" of the transverse axis is located.

v. The second record is placed on the articulator and the subsequent apparant transverse axis is obtained.

The interocclusal records are considered verified if the pointers intersected with the condylar housings at the same point. If a kinematic facebow was used, this point must be the articulator transverse axis.

The Buhnergraph has also been utilized in research to evaluate various methods of obtaining CR (Lundeen, 1974). It must be realized that only a limited number of parameters of transverse axis positional alteration can be detected with the Buhnergraph (Fattore, 1979). Lateral motions of the axis do occur during interocclusal record procedures (Goasso and Sharry, 1968). Such displacement will not be detected by the Buhnergraph. Therefore, the Buhnergraph is a limited verification instrument that deals with less displacement parameters than split cast technique and locates only the intercepts of the trans- 
verse axis as it passes through the condylar housings.

Remien, et al (1974) altered a Denar articulator to produce a device similar to the Buhnergraph. In addition to the condylar housing measurement, three-dimensional movement of the incisal pin was also taken under consideration. Also, unlike Lundeen, Remien treated the value obtained on the condylar housing as a displacement and subsequently reported both the horizontal and vertical components of intercept displacement. Remien's methodology yielded results that were not integrated in that only the raw data obtained from the various measuring areas were reported.

Ironically, one of the most "advanced" devices to be conceived for analysis of mandibular displacement was designed more than 20 years ago by a pioneer in mandibular physiology. Posselt's "Gnathothesiometer" (1957) is a very comprehensive device for obtaining the displacement of casts caused by wax bite registrations. The device is similar in design to Remien's modified Denar articulator. In addition to Remien's measurements, the gnathothesiometer has a specific provision for intercondylar distance and even "condylar breadth" (as determined by radiographs). Posselt, like Remien, only reports raw data from his device, as opposed to integrating it into descriptive refined data of mandibular displacement.

The final displacement analysis device to be discussed is a modified Ney articulator (Hoffman, et al, 1973). This device was designed to evaluate articulator condylar position resulting from various clinical centric relation recording procedures. The measurement technique was accomplished by "boxing out" the condylar housings 
into open, hollow cubes. The distance between the articulator condylar sphere and the appropriate reference wall of the modified condylar housing was determined by using feeler guages. The resulting data yielded full description of articulator condylar displacement, but offered no indications of axial rotation of the articulator's transverse axis.

In conclusion, several devices have been designed to evaluate mandibular position in space. Although many approach the measurement of all data necessary for fully describing displacement, there seems to be no entirely descriptive or common format for any of the final experimental results. 


\section{CHAPTER III}

MATERIALS AND METHODS

PART 1:

Theoretical Considerations and

Instrument Design Specifications

Derivation of a Standardized Format for Experimental Data.

The major objective of this study is the development of an instrument that will provide fully descriptive and meaningful data for the evaluation of articulator mounting procedures. It would also be desirable for the data to be in such a form as to permit easy manipulation for modelling and comparison with other investigators. A secondary purpose is the execution of a simple experiment using such an instrument. Before either of these goals can be realized a descriptive format for measurement, refinement, manipulation, presentation, and evaluation of the data must be formulated. Once this is accomplished, various designs of instruments can be evaluated within the constraints of the desired output parameters. Finally, utilization of the instrument along with a rational descriptive format will assist the reader in conceptualizing the types of error and displacement that do occur. Also, a logical format of displacement analysis could possibly contribute to an improvement in the standardization of data formats for future investigations. 
The dental literature offered no format that met the requirements of this study. The methodologies of Abler (1972), Messerman, et al (1970), and Lundeen, et al (1970) were fully descriptive of mandibular displacement. However, the data formats used did not allow for either easy conceptualization (the fact that a playback simulator was necessary in two of the studies exemplifies this) or comparison among the investigators. Kinzel's (1972) format utilizing the screw axis allowed for the added complexity of body movement, a consideration not important in this investigation.

As discussed previously, there are several methods of evaluating the position of a solid in space. This study utilizes two descriptive systems that operate in tandem and whose data are easily interchangeable. The measurements of mandibular displacement and the final output parameters are presented using a global-local system. Additionally, pure point displacements of three or more arbitrarily chosen points on the solid (in this case the solid is one member of the articulator in reference to the other) are also provided for the sake of modelling and simulation. A simple application of mathematical modelling is used to demonstrate hypothetical condylar displacement occuring from interocclusal distortions.

Application of the Global-local Reference System.

The mandibular member of a modified Whip-mix articulator was chosen to be fixed. The maxillary member was therefore "floating" in reference to the mandibular member. The origin of the coordinate axes was placed at the midpoint of the imaginary line connecting the centers of the left and right condylar spheres. This line also defines 
the $Y$-axis. The $X$-axis is perpendicular to the $Y$-axis in the hor $i-$ zontal plane at the origin. Therefore, the $x$-axis is synonomous with the anterior-posterior axis of the mandibular member. Finally, the $z-$ axis is perpendicular to the XY plane at the origin. A similar local coordinate system was assigned to the maxillary member of the floating articulator. The $x$-axis (note: references to the global axes are in capital letters and references to the local axes are in noncapitalized print) is defined by the inter-condylar housing axis. The $y$-axis is synonomous with the maxillary member's anterior-posterior axis, and the $z$-axis is perpindicular to the $x y$ plane. The intersection of the $x, y$, and $z$ axes defines the local origin. This point is also the intercondylar housing axis midpoint, or intercondylar point (henceforth referred to as ICP).

In the absence of an imperfect mounting, the global and local coordinate axes are coincident. Therefore, any distortions brought about by inaccurate interocclusal record procedures results in a noncoincidence of the two coordinate systems. This is synonomous to a distortion in articulator transverse axis position. In that the articulator transverse axis is an extremely important entity in terms of conceptualization of interocclusal relationships, this global-local system will be highly conducive to formatting meaningful results. In fact, the displacement of the local (maxillary) coordiante system in reference to the global (mandibular) coordinate system can be fully expressed by the overall displacement of ICP in conjunction with the familiar aeronautical terms of pitch, yaw, and roll (Figure 1) applied to the $y$-axis (syn. articulator transverse axis) as follows: 

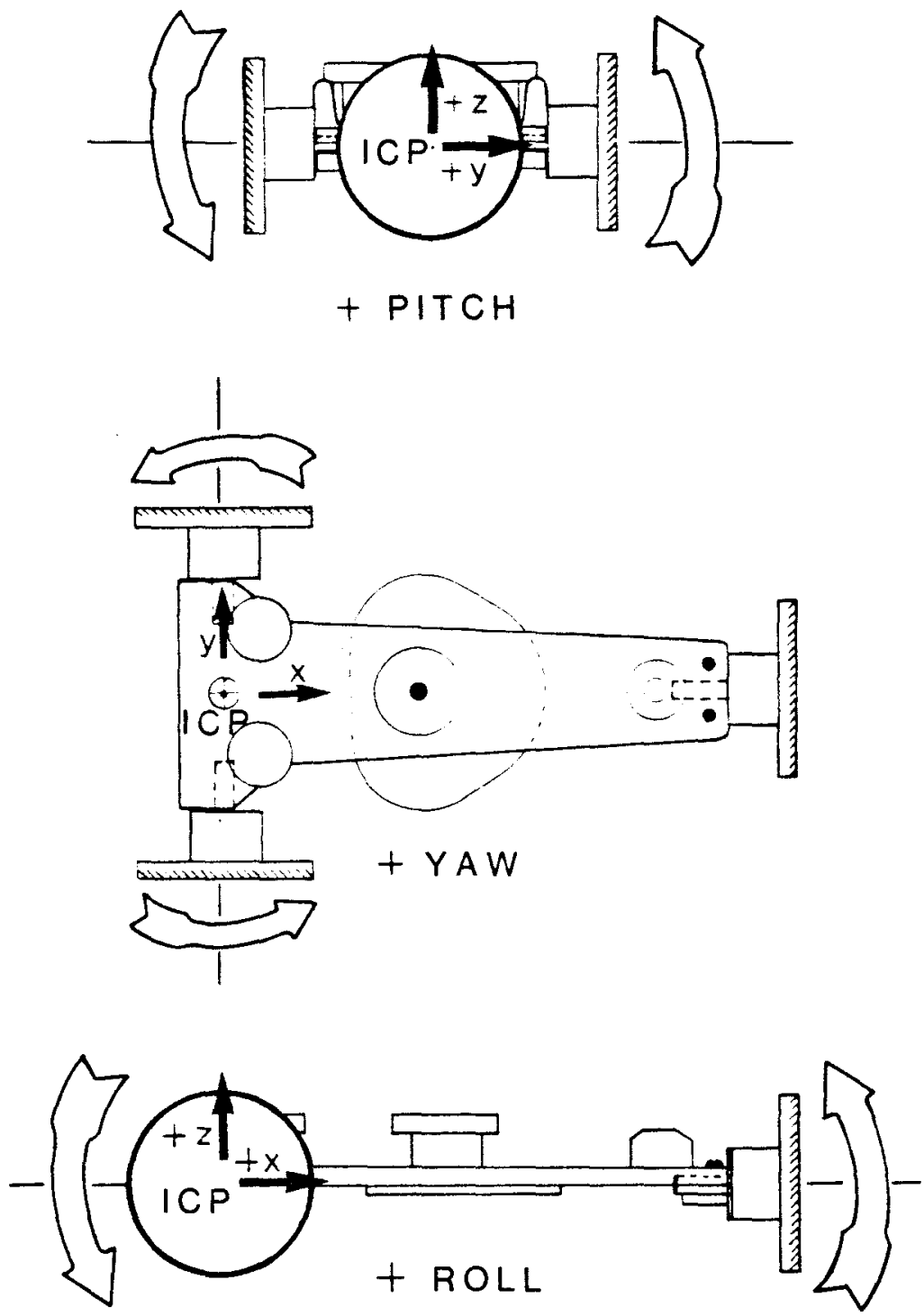

Figure 1: Diagrammatic Demonstration of Pitch, Yaw, and Roll as Applied to the Maxillary Assembly 


\section{PARAMETER:}

$x$ displacement

y displacement

$z$ displacement

Pitch

Yaw

Rol 1
ANATOMICAL EQUIVALENT:

pure anterior-posterior displacement of ICP.

pure left-right displacement of ICP.

pure superior-inferior displacement of ICP.

transverse axis rotation in the coronal plane.

transverse axis rotation in the horizontal plane.

pure rotation of the transverse axis.

Additionally, four arbitrary points were chosen on the maxillary member of the articulator as follows:

Point 1

Point 2

Point 3

Point 4 maxillary intercondylar point (ICP). intersection of maxillary articulator member's principle casting's perimeter with the transverse axis (left side). intersection of casting's perimeter with transverse axis (right side). intersection of casting's perimeter and anterior-posterior axis (anterior).

The three-dimensional coordinates of these four points was determined for the pre-experimental situation. The resulting coordinate triplets were placed into an array of $3 \times 1$ matrices. Such a format resulted in the ability to define the position of the maxillary member relative to the global Cartesian coordinate system. Secondly, the coordinate matrix format allowed for the more extensive manipulation of the data through the utilization of transformation matrices (Rogers and Adams, 1976; Roedema, 1978). Thus extrapolation of the data to produce numerical values for the displacements of any point on the articulator was 
made possible. This ability is potentially useful in that the data can be potentially presented in terms of, for example, left/right condylar displacement, anterior pin displacement, arbitrary cast landmark displacement, or even cusp-tip displacement.

Thus two data formats were chosen for the use in this study. A global-local origin-axes displacement system was formulated for the sake of fully describing displacements of the maxillary member of a Whip-mix articulator in reference to the mandibular member. It will be demonstrated that this format is applied to the measurement technique utilized. Secondly, a pure Cartesian point displacement format will be used concurrently to allow for the manipulation of data by transformation matrices. This will permit the experimenter to calculate the three-dimensional displacements of any chosen points on the articulator.

Instrument Design and Construction Specifications.

The instrument constructed allowed for the six-degree-of-freedom measurement of displacement between maxillary and mandibular members of an articulator by detecting the three-dimensional displacement of ICP along with the angular displacements of the articulator transverse axis and the articulator anterior-posterior axis. For descriptive purposes, the instrument can be considered to consist of three interacting components: (1) the maxillary assembly, (2) the base assembly, and (3) the optical system.

1. The maxillary assembly.

The maxillary component of the device is a modified maxillary 
member of a Whip-mix articulator (Figure 2). The main, "T-shaped" portion (henceforth referred to as the "casting") was used as a foundation for the assembly. The plaster-cast plate retaining screw and housing brackets were left intact. An anterior-posterior 1/2-circular groove was machined into the inferior surface of the casting (Figure 3). The maximum height of the groove was $3.175 \mathrm{~mm}$, the same as the manufacturer's inter-condylar housing groove. The heights of the curverature of the anterior-posterior groove and the intercondylar housing grooves intersect. This intersection defines the location of the intercondylar midponit (ICP). An $11 \mathrm{~mm}$ viewing port was drilled, perpindicular to the casting, at the ICP (Figure 4).

The condylar housings were removed, rendering the maxillary casting as a floating member. Each housing was replaced by a firstsurface mirror assembly (Figures $5 \& 6$ ). Each mirror assembly consisted of a $16 \mathrm{~mm}$ (radius) $\times 4.8 \mathrm{~mm}$ mirror (surface flat to $2.54 \times 10 \mathrm{E}-4 \mathrm{~mm}$ ) epoxied to an arbor. The arbor facing is $22.4 \mathrm{~mm}$ in diameter and perpindicular to (and centered with) the arbor shaft. The arbor body is $14.4 \mathrm{~mm}$ long and has a rear facing parallel to the front facing. The shaft is $15 \mathrm{~mm}$ long and $6.350 \mathrm{~mm}$ in diameter. The arbor shaft diameter is identical to the diameter of the former condylar housing's shaft. The mirror assemblies, therefore, fit into the maxillary casting in lieu of the condylar housings. The manufacturer's condylar housing clamp plates and their associated hardware were used, unmodified, to retain the mirror assemblies. Since the center of the condylar housing shaft is coincident with the maxillary member's transverse axis (personal communication with staff at Whip-mix Corporation), the central 


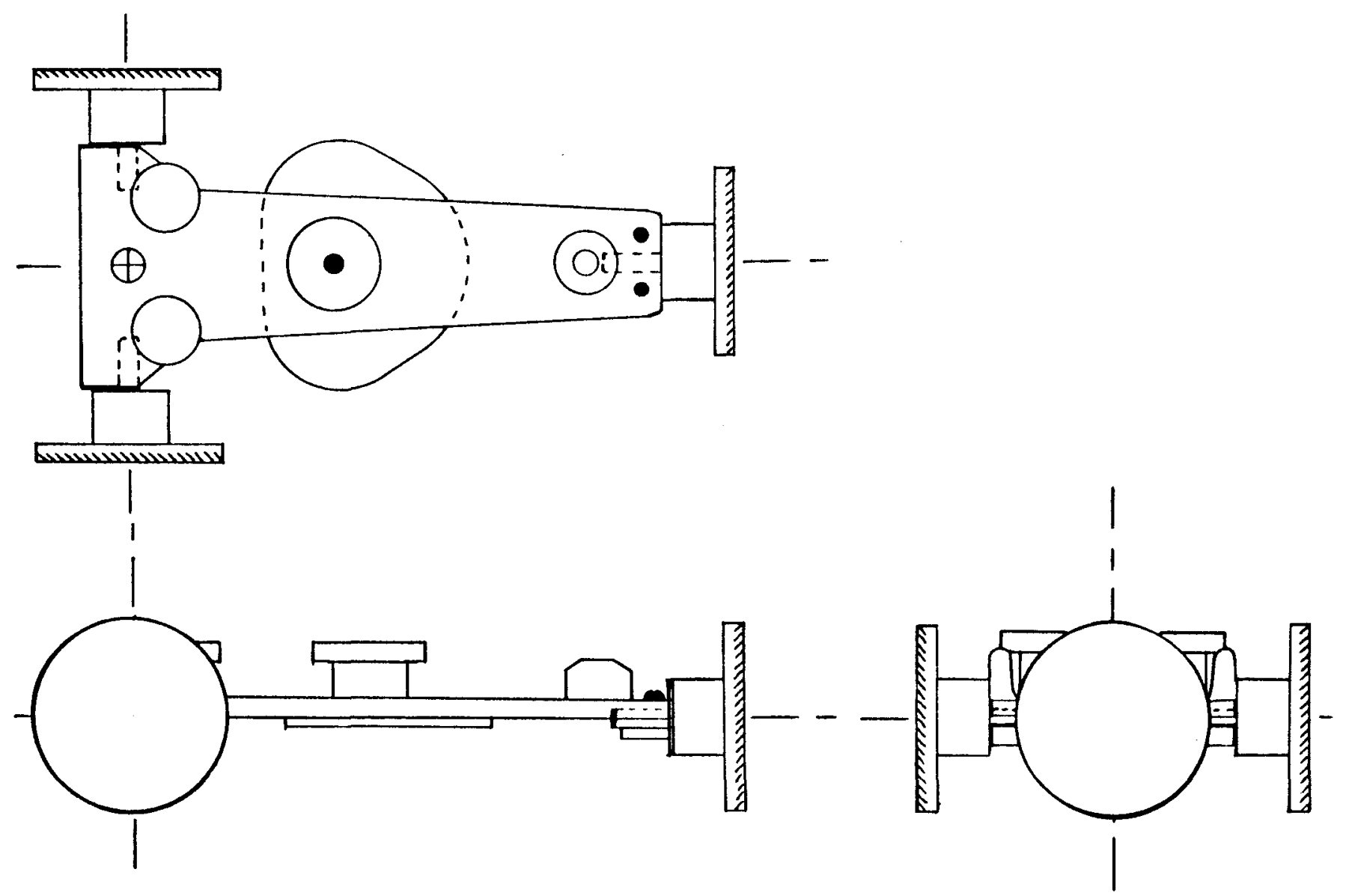

Figure 2: Modified Maxillary Member of Articulator 


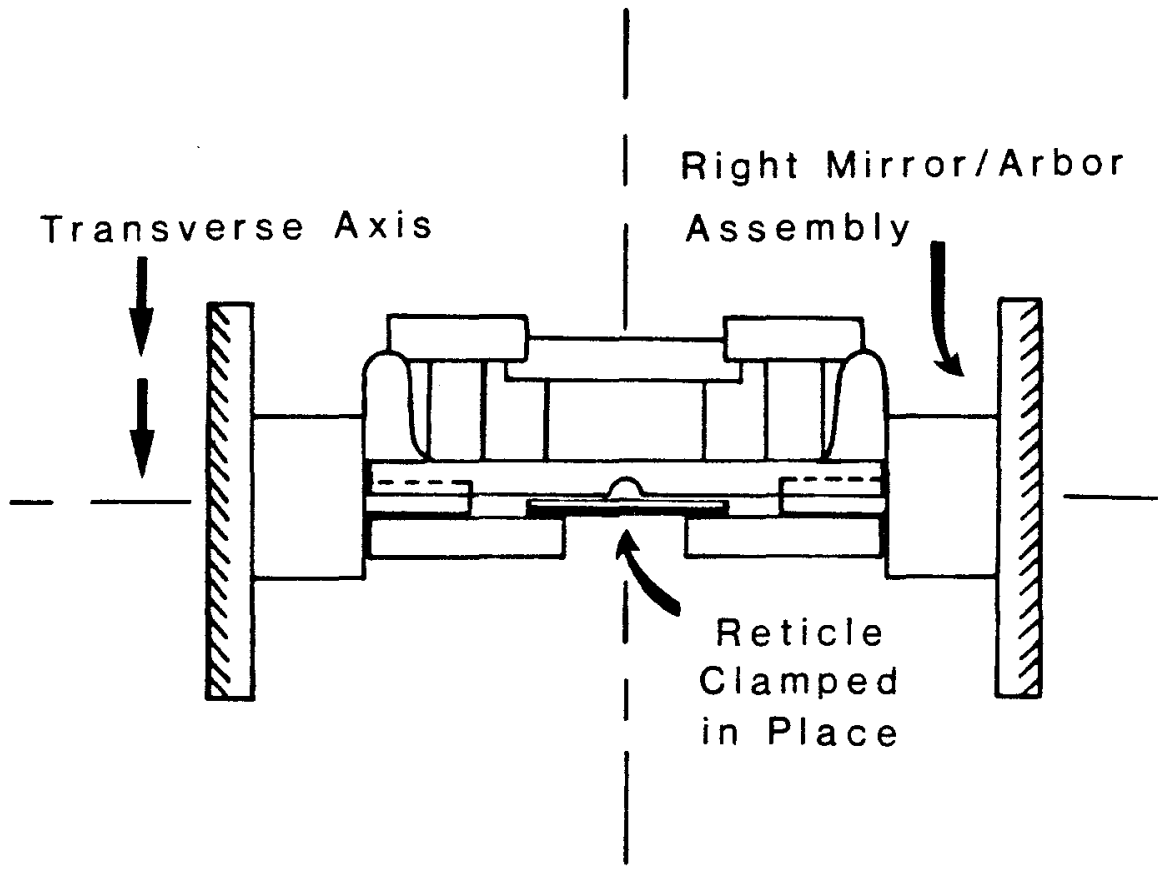

Figure 3:

Posterior View of Maxillary Assembly 


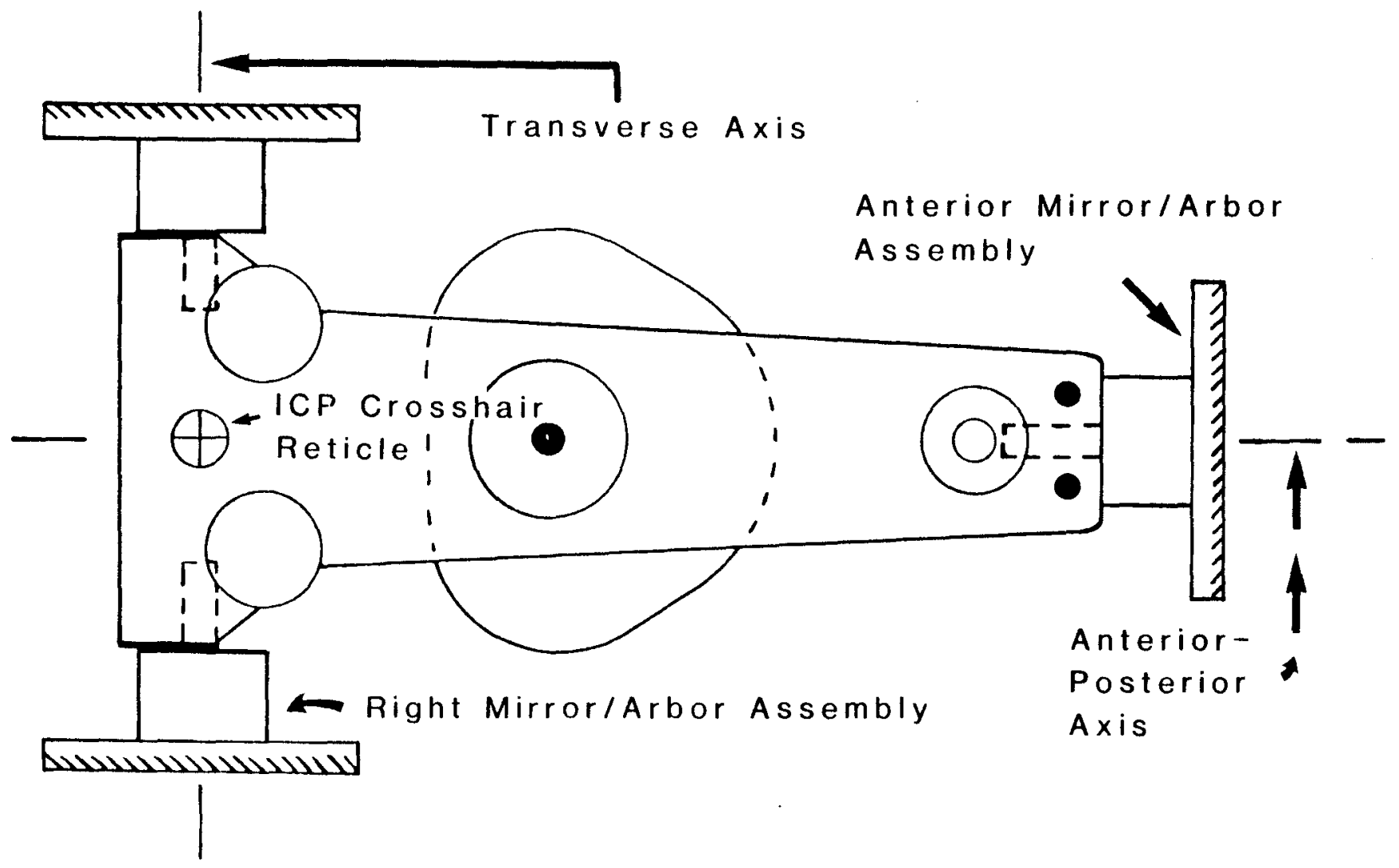

Figure 4: Superior View of Maxillary Assembly 


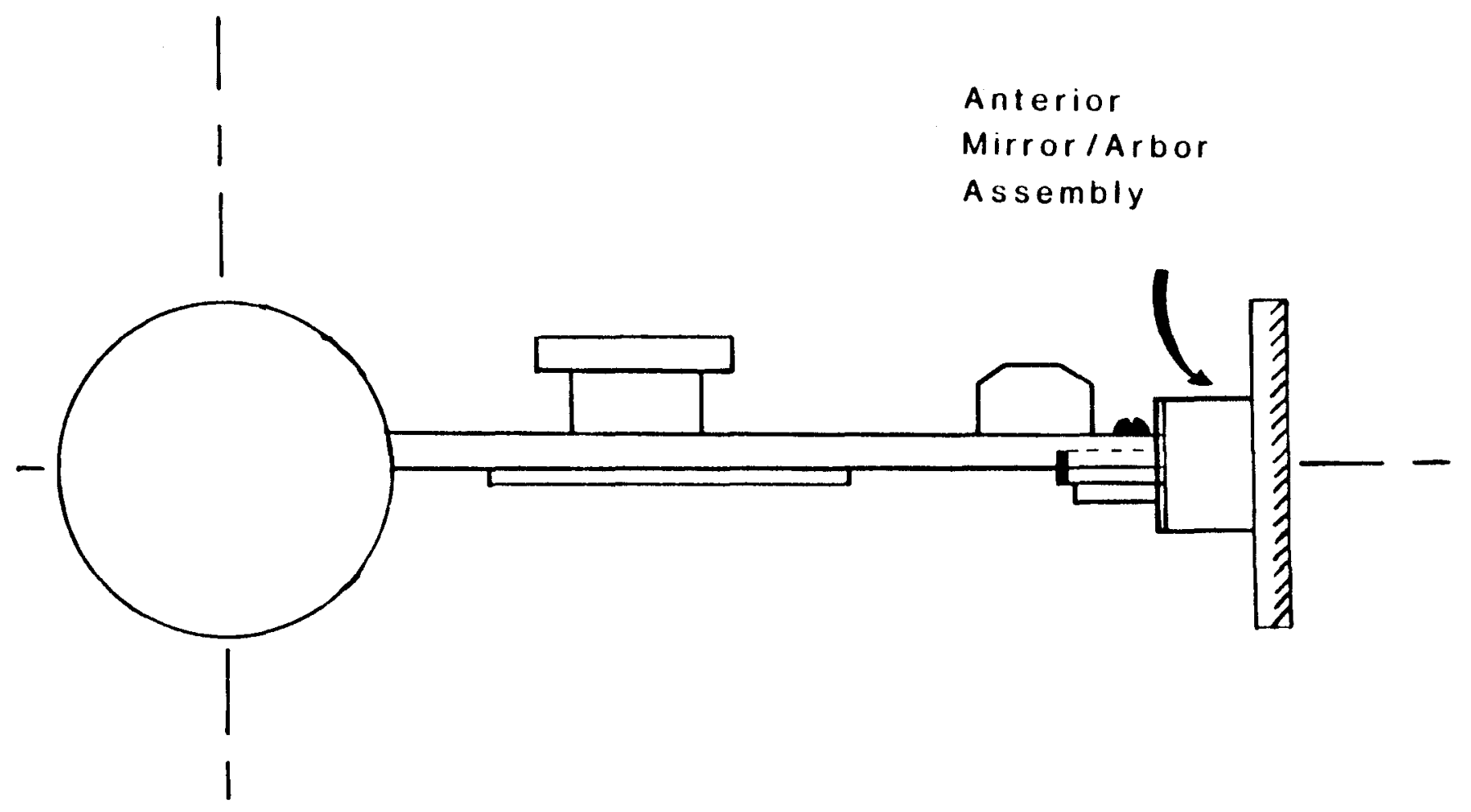

Figure 5:

Right Side View of Maxillary Assembly With Right Mirror Removal 


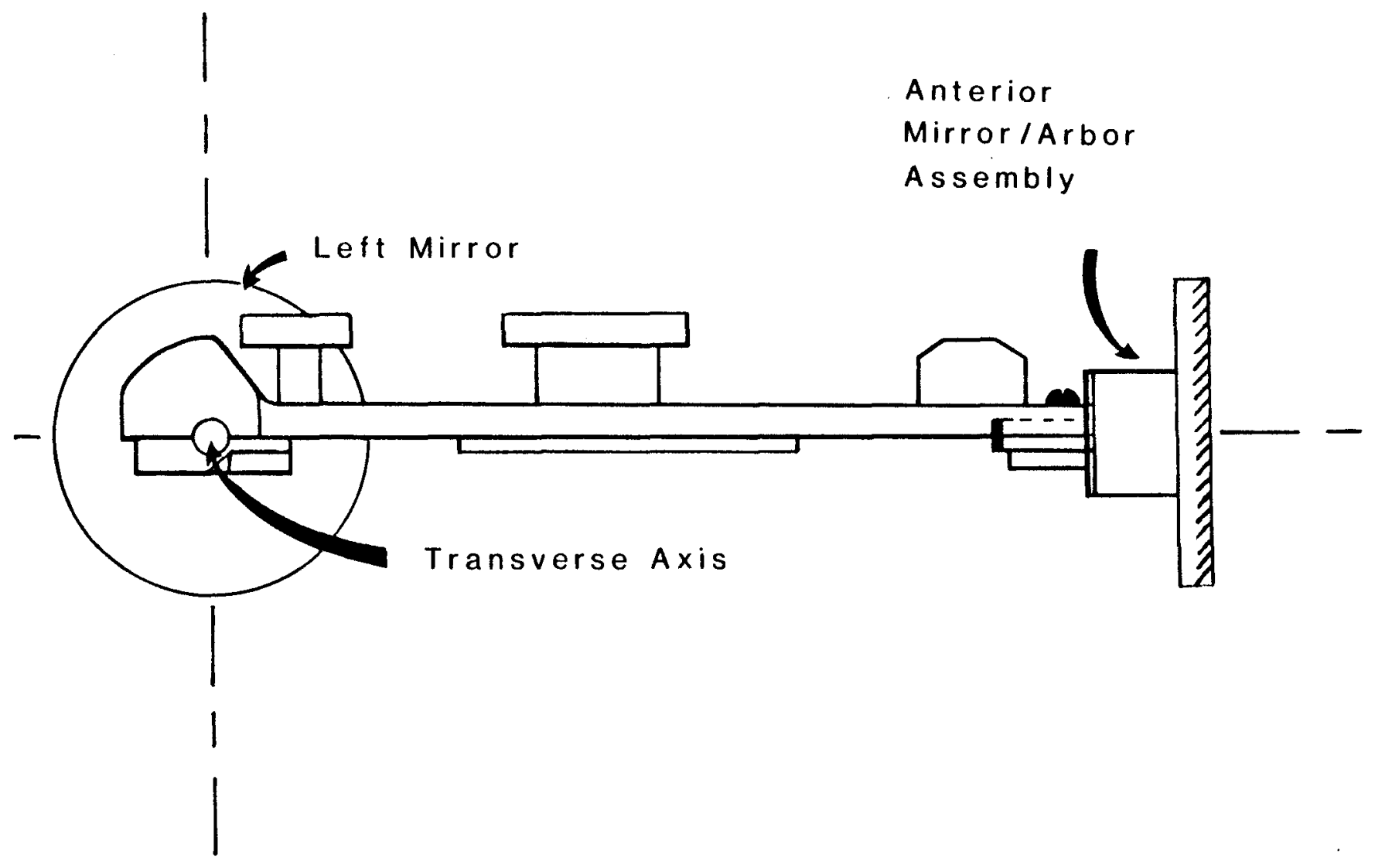

Figure 6:

Right Side View of Maxillary Assembly With Right Mirror in Place 
optical axis of the mirror assemblies are coincident with the transverse axis. Additionally, their surfaces are perpindicular to the transverse axis of the casting.

The condylar housing clamps were also employed to retain a $20.8 \mathrm{~mm}$ (diameter) $\times 1.9 \mathrm{~mm}$ glass microscope reticle (figure 3 ). The reticle possesses a first-surface crosshair. The plane of the reticle's first surface is coplanar with the plane defined by the transverse and anterior-posterior axes of the maxillary casting. The reticle is clamped and epoxied in such a position as to render the cross-hair intersection coincident with the ICP. The ICP is, then, visable through the $11 \mathrm{~mm}$ viewport.

Finally, a third mirror assembly, identical to the others, was mounted to the casting at its most anterior border (Figures 4 \& 7 ). A flat clamp retained by two $6 / 32$ (English) screws was fabricated to affix the arbor shaft into position in the anterior-posterior groove of the casting. Thus, the anterior mirror's optical axis is colinear with the anterior-posterior axis of the maxillary casting. Therefore, the optical axes of the left and right condylar mirror assemblies intersect with the optical axis of the anterior mirror assembly at the intercondylar point. The inter-condylar point is coincident with the crosshairs of the reticle. Two blueprint-style drawings are provided for visualization of the maxillary assembly (figs. $7 \& 8$ ).

\section{The base assembly.}

The base assembly consists of two major components: (1) the mounting table (fixed component), and (2) an array of microscopes and dial guages (movable component). 


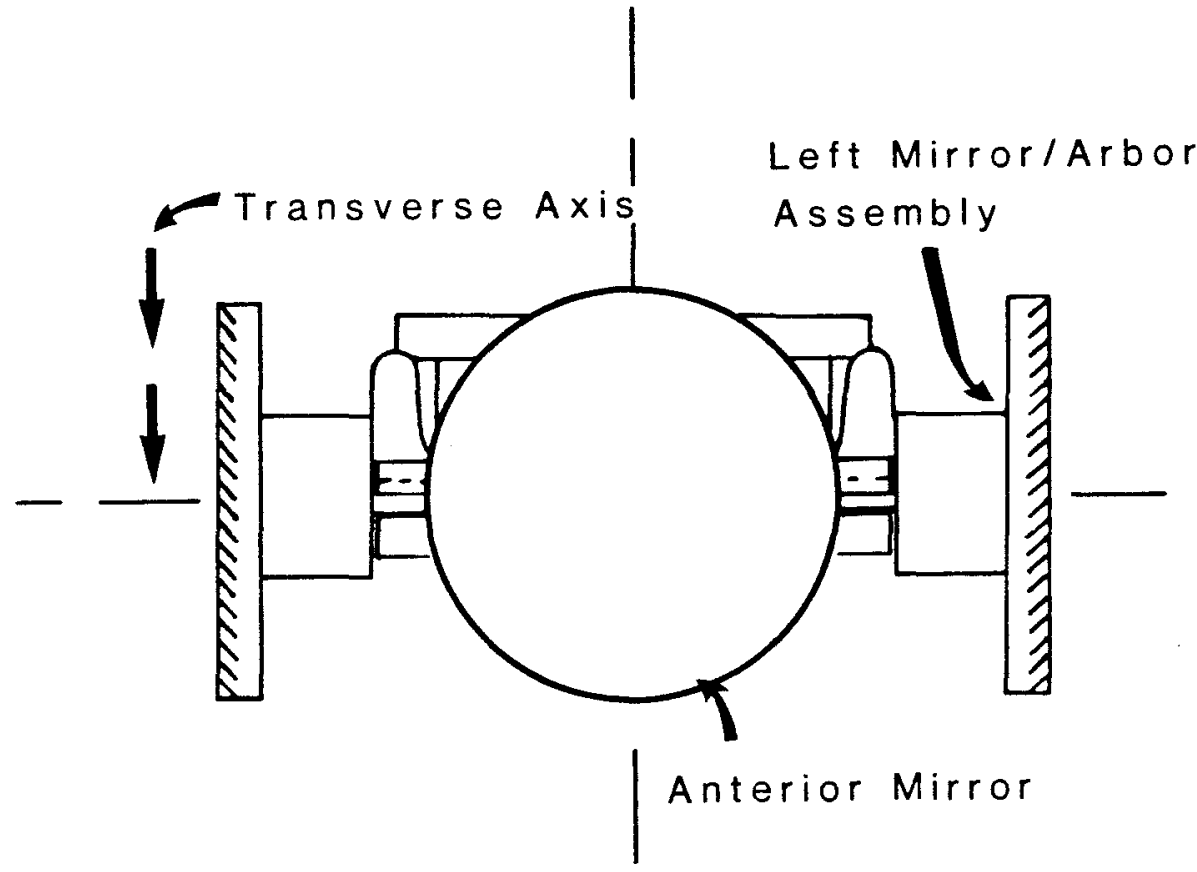

Figure 7:

Anterior View of Maxillary Assembly 


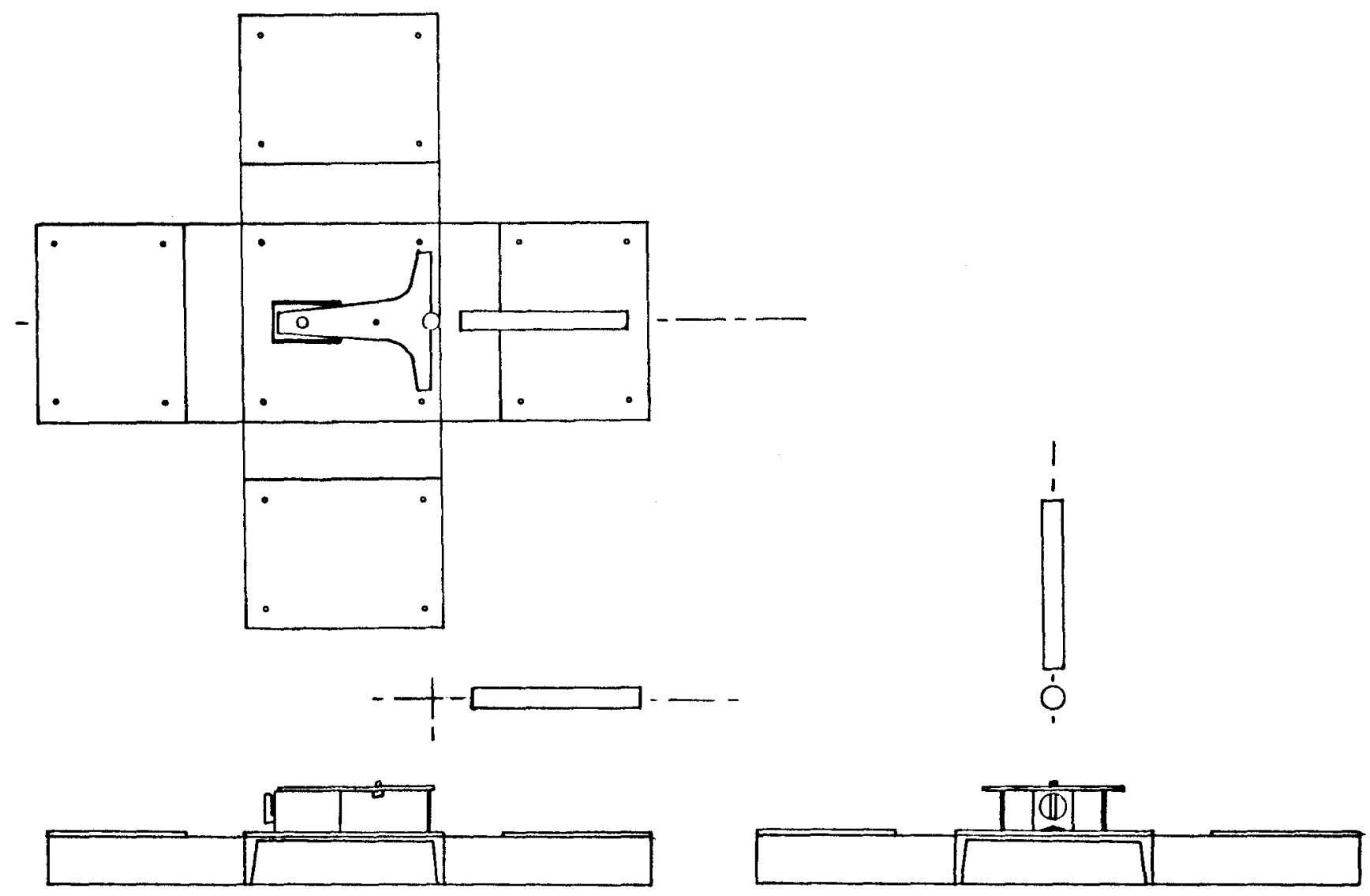

Figure 8: Base Assembly 
The mounting table consists of five machined steel plates mounted to a steel superstructure (fig. 9). The superstructure is constructed with $200 \mathrm{~mm} \times 52 \mathrm{~mm}$ channel iron. The thickness of the steel ranges from $5 \mathrm{~mm}$ to $15 \mathrm{~mm}$ (figs. $10 \& 11$ ). Two $200 \mathrm{~mm}$ lengths of channel iron were welded to a $600 \mathrm{~mm}$ length of channel iron to form a "Swiss cross" arrangement. The surface of this assembly was ground to a roughness height of $.13 \mathrm{~mm}(.005$ inches $)$. Mounting holes were drilled so as to allow the positioning of 5 steel plates on the channel iron. The center plate $(200 \mathrm{~mm} \times 200 \mathrm{~mm})$ was mounted at the intersection of the four legs of the cross. Each of the four peripheral plates $(200 \mathrm{~mm}$ $X 150 \mathrm{~mm} \times 7.9 \mathrm{~mm})$ was positioned on one of the four legs of the cross. Each of the 5 plates was secured by four $6.35 \mathrm{~mm}$ (diameter) fine-threaded bolts. The 5 plates were rendered parallel by placing shims $(5.0$ X 10E-04 inches) at appropriate locations before final tightening of the bolts. The surface of each plate was ground, honed, polished, then lapped to a roughness height of $1.2 \times 10 \mathrm{E}-02 \mathrm{~mm}$.

An adjustable array of instrumentation was mounted on the mounting table by means of magnetic mounting units*. Each mounting unit had a surface honed to a roughness height of $2.4 \times 10 \mathrm{E}-02 \mathrm{~mm}$. The contact of the bases' honed surface with the mounting table's lapped surface allowed for uniplanar positioning of any device affixed to a magnetic base, no matter what the location of the base.

The horizontal component of the mandibular member of a Whipmix articulator was affixed to a magnetic base at the incisal guide

*Yausa International Inc., Carlstadt, N. J. 


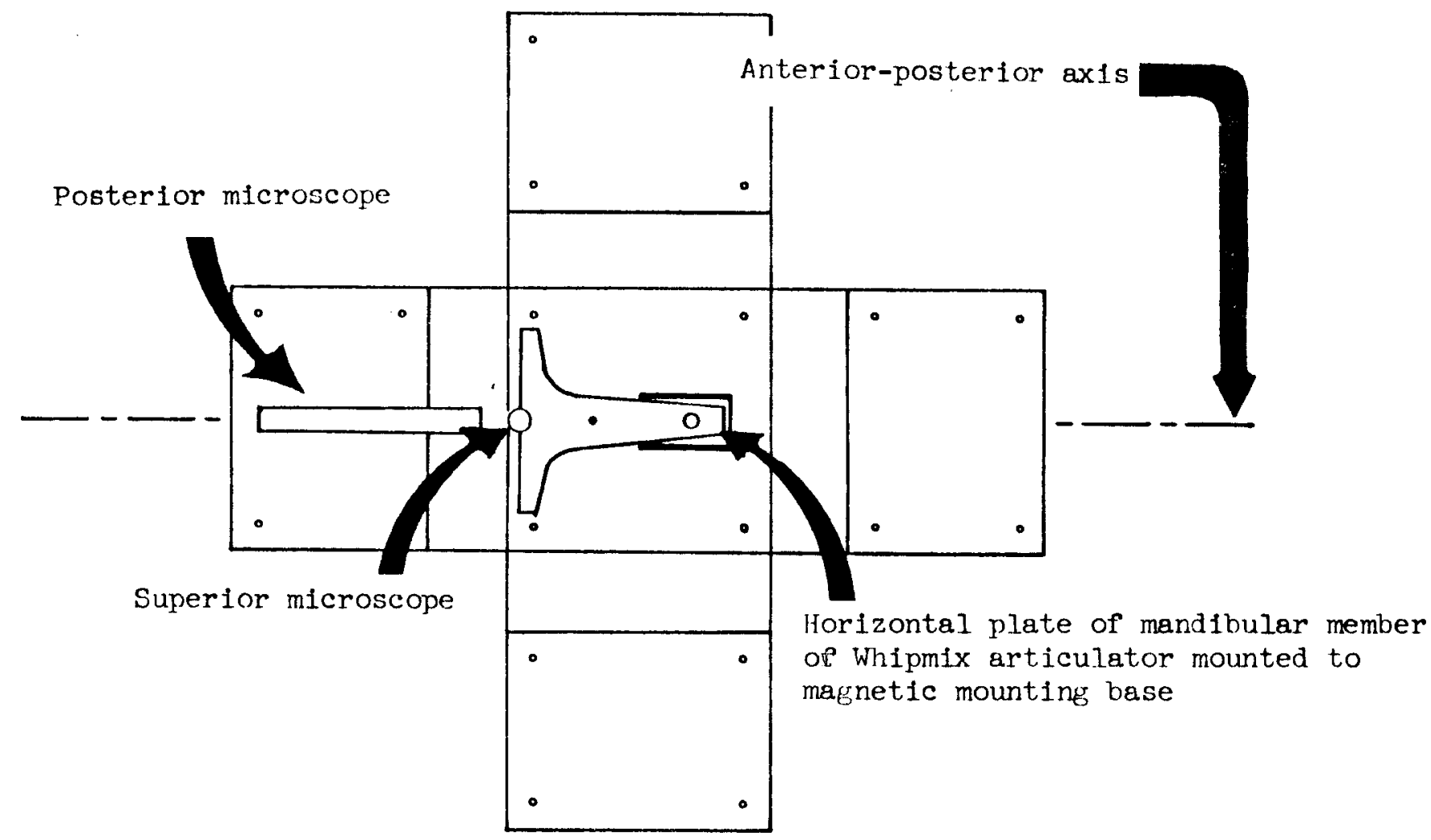

Figure 9: Superior View of Mounting Table 
Anterior-posterior axis
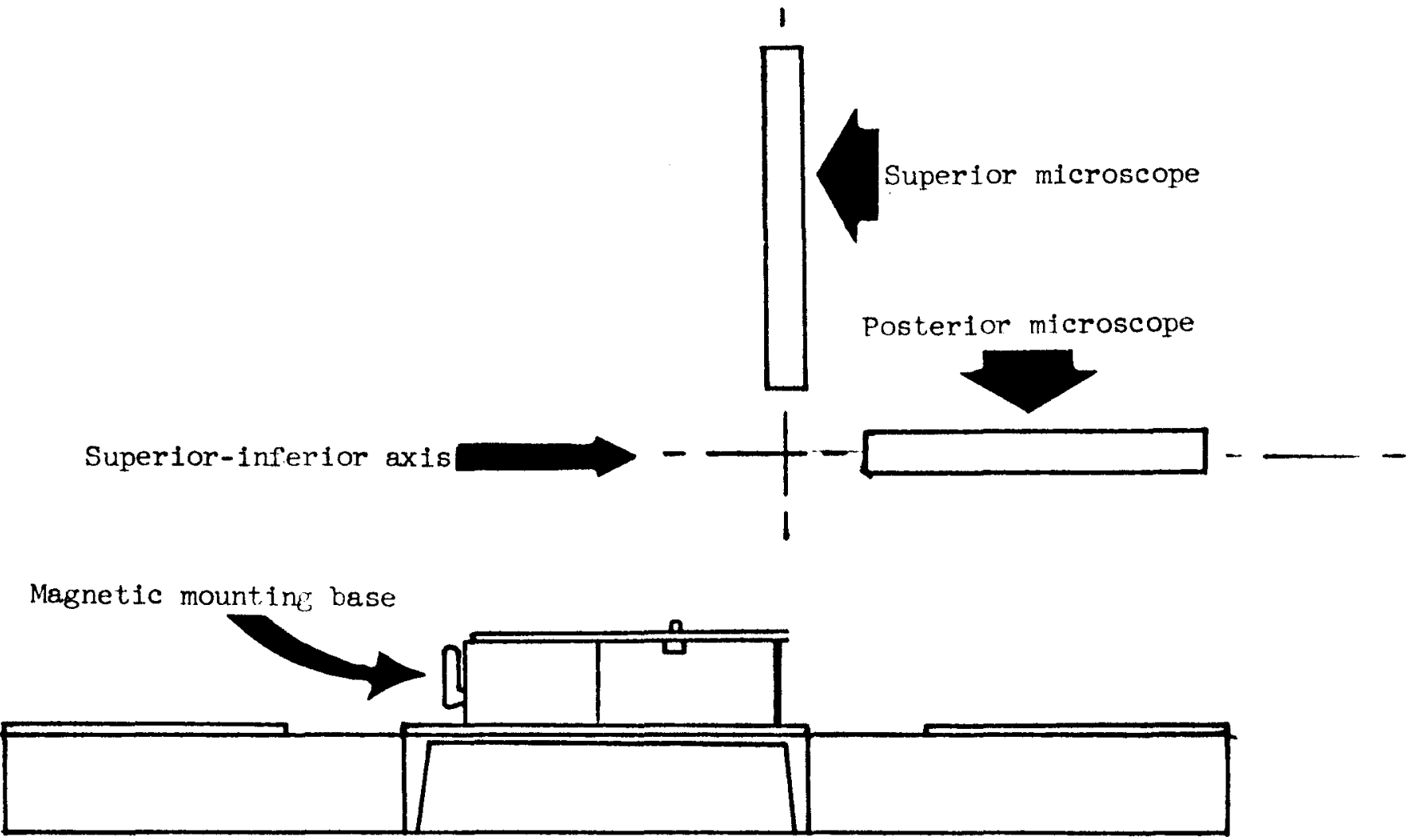

Figure 10: Lateral View of Mounting Table 


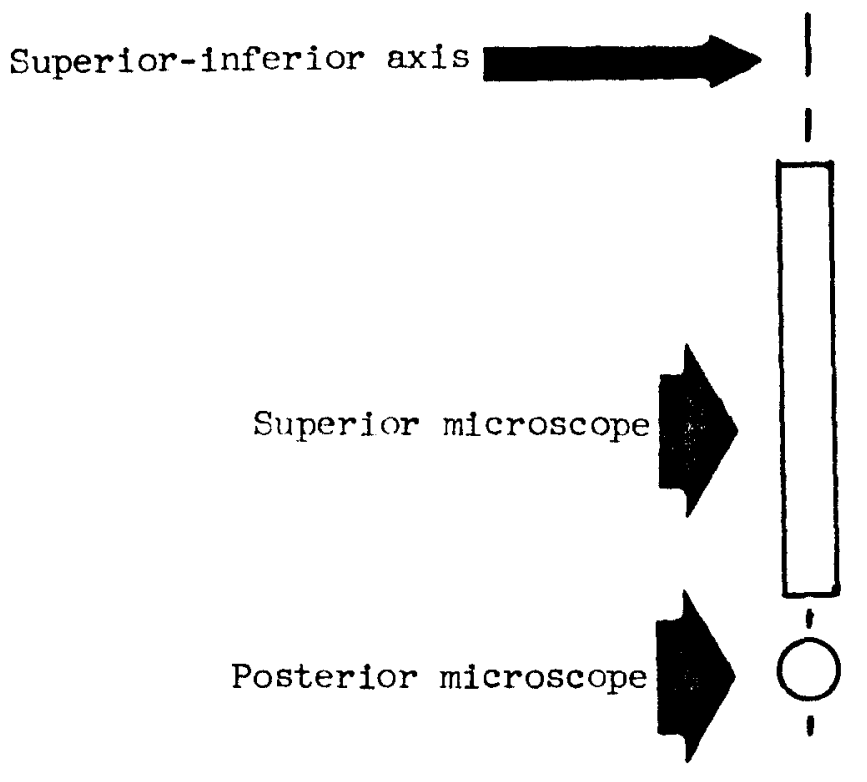

Lapped steel plate for consistent inounting of

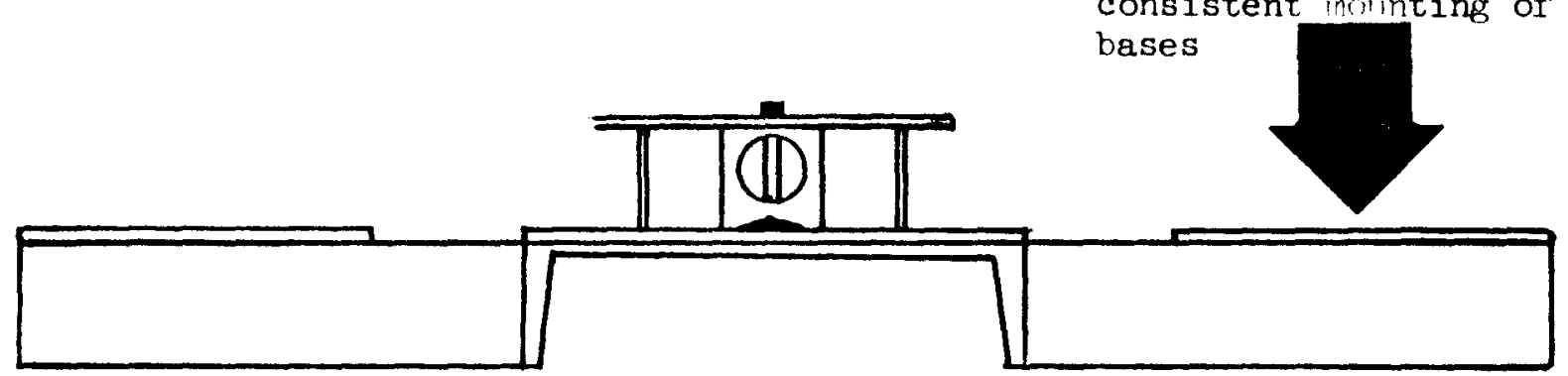

Figure 11: Anterior View of Mounting Table 
table slot. The entire condylar support superstructure was removed and two bolts were screwed into the articulator's leveling taps (from the inferior aspect) so as to provide support to the posterior area of the aluminum plate. This assured that the mandibular assembly would remain stable during any manipulation. The mandibular plastercast plate retaining screw was left intact in its original position.

An array of 4 magnetic bases was situated to support 2 microscopes and 3 dial guaçes (fig. 12). Each microscope contained a crosshair measuring reticle. Each dial guage was calibrated in units of $2.54 \times 10 \mathrm{E}-02 \mathrm{~mm}(1.0 \times 10 \mathrm{E}-02$ inches $)$. A11 components were mounted to the magnetic bases by means of various rods, adjustable rod clamps, and castings. The microscopes were positioned so as to view the ICP area from the superior aspect and the posterior aspect. The ICP area is considered to be the approximate location of ICP when the maxillary assembly is related to the mandibular assembly by means of dental casts with or without interocclusal record materials. Each microscope was mounted in a rack and pionion assembly. Each rack and pinion was in turn mounted on a casting that was guided by two rods that were parallel with each other and the optical axis of the microscope. Movement of this casting along the parallel rods allowed for focusing of the microscope without lateral deviation of its optical axis. The superior microscope was movable in the anterior-posterior and mediolateral directions by means of its rack and pionion. This allowed positioning of the microscope so as to observe overlapping of the microscope reticular crosshair with the maxillary assembly reticle crosshair. The amount of microscope displacement realized by such 


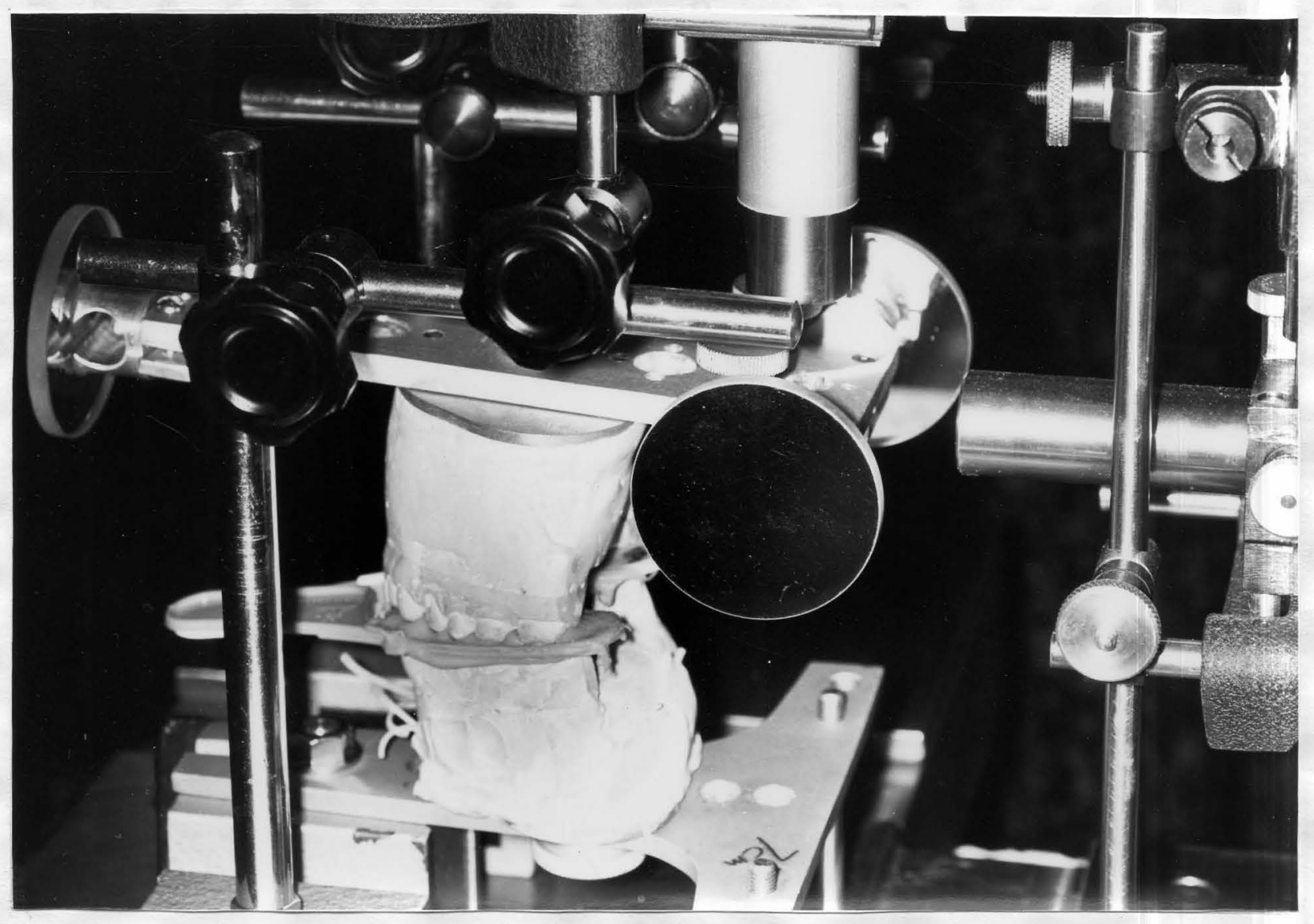

Figure 12: Photograph of Experimental Setup 
maneuvering is detected by two dial guages engaging the appropriate surfaces of the rack and pionion assembly. The posterior microscope was movable in the superior-inferior and medio-lateral directions. This microscope was set up scpedifically to determine the position and/ or displacement of ICP in the superior-inferior direction. Mediolateral movement of the posterior rack-and-pinion permitted the experimenter to center (medior-1aterally) the microscope in respect to the maxillary assembly. The superior-inferior movement allowed the experimenter to seek the level of the ICP. A third dial guage engaged the rack-and-pinion of the posterior microscope so as to detect the superior-inferior position and/or displacement of the ICP.

Therefore, a highly machined (lapped) mounting table was constructed to support machined magnetic bases in coplanar orientation. These bases, in turn, supported (1) a modified mandibular member of a Whip-mix articulator and (2) an array of 2 measuring microscopes mounted in two-dimensional racks and pionions, and 3 dial guages. When the maxillary assembly is related to the mandibular assembly by means of dental casts, the experimenter is able to seek the three-dimensional location of the ICP reticle by observing the microscope crosshairs while manipulating the rack-and-pinion knobs. The anteriorposterior $(x)$ and medial-lateral $(y)$ position of ICP is detected by horizontal dial guages engaging in the superior microscope. The superior-inferior ( $z$ ) position of ICP is detected by a vertical dial guage engaging the posterior microscope.

3. The optical system.

The remaining th ee parameters of displacement pertain to the 
angular movements of the maxillary assembly's coordinate system (local) relative to the mandibular assembly's coordinate system (global). This can be better visualized by recalling that we can choose one of the three axes of the local coordinate system as an axis of orientation and assign the familiar terms of "pitch, yaw, and roll" to its angular displacement. The axis of orientation will be the transverse axis. Therefore, skew of the transverse axis parallel to the frontal plane is analagous to "pitch" while skew of the transverse axis parallel to the horizontal plane is analagous to "yaw." "Roll" is synonymous to rotation of the maxillary assembly about the transverse axis (fig. 1). This is also equivalent to skew of the anterior-posterior axis in the saggital plane.

Two slide projectors were set up with the projections of their optical axes in the global XY plane perpindicular to one another (fig. 13). The projection of each optical axis in the appropriate vertical plane was at a known angle (fig. 14). This allowed each projector to be aimed at one of the maxillary assembly's first-surface mirrors (in the non-displaced condition) in such a manner as to effect a three-way intersection of the projector's optical axis, the mirror's optical axis, and the plane of the mirror's first surface. A first-surface mirror was chosen for use because first-surface mirrors have the advantage of reflecting 1 ight without any accompanying refraction caused by the mirror's bulk of glass (Smith and Cooper, 1972). Each projector was a known distance from the main apparatus (2 to 3 meters) and projected the image of a glass cross-haired reticle onto the appropriate mirror. The image was subsequently reflected back to a wall positioned behind 


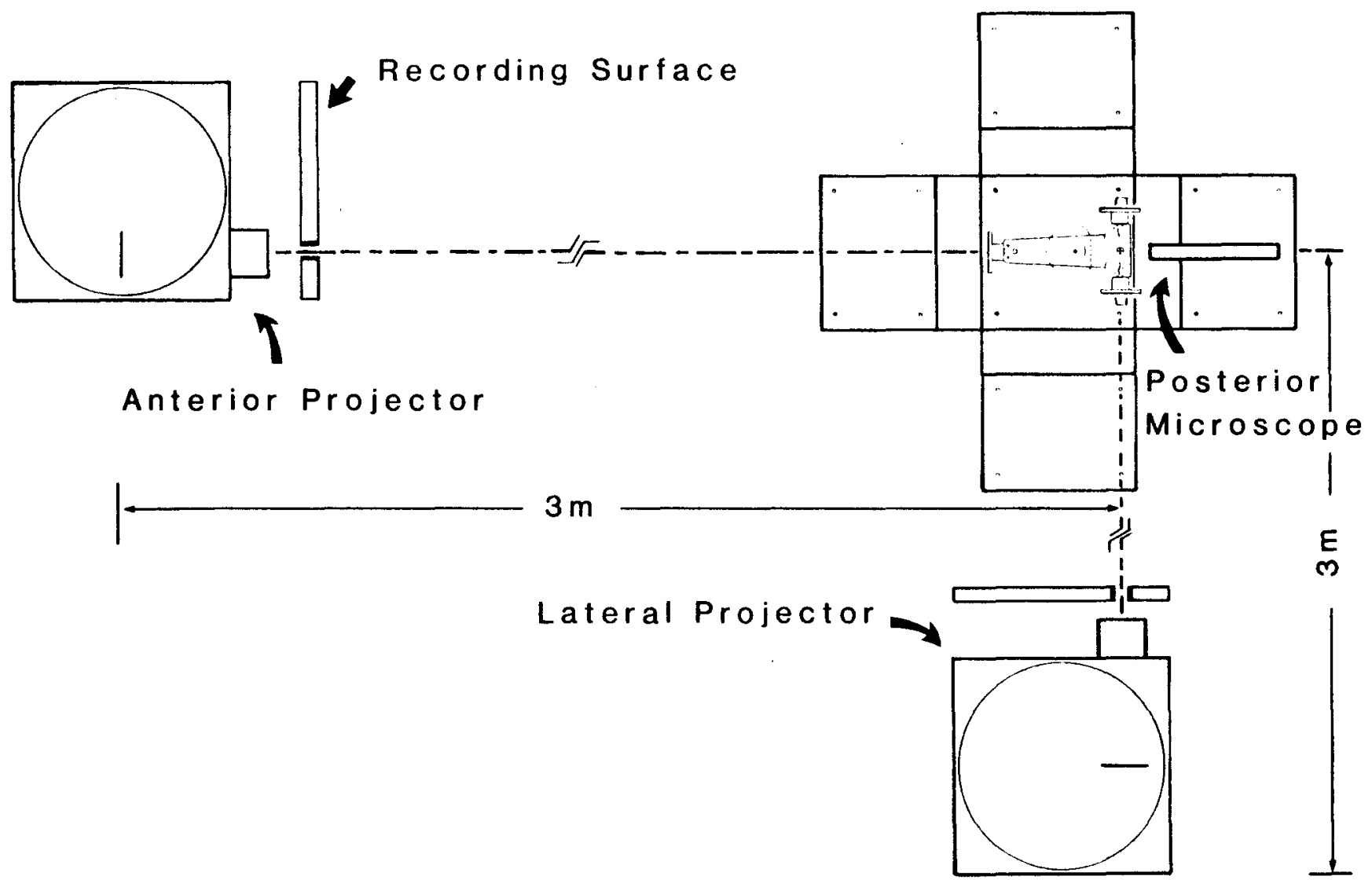

Figure 13:

Diagrammatic View of Apparatus from Superior Aspect 


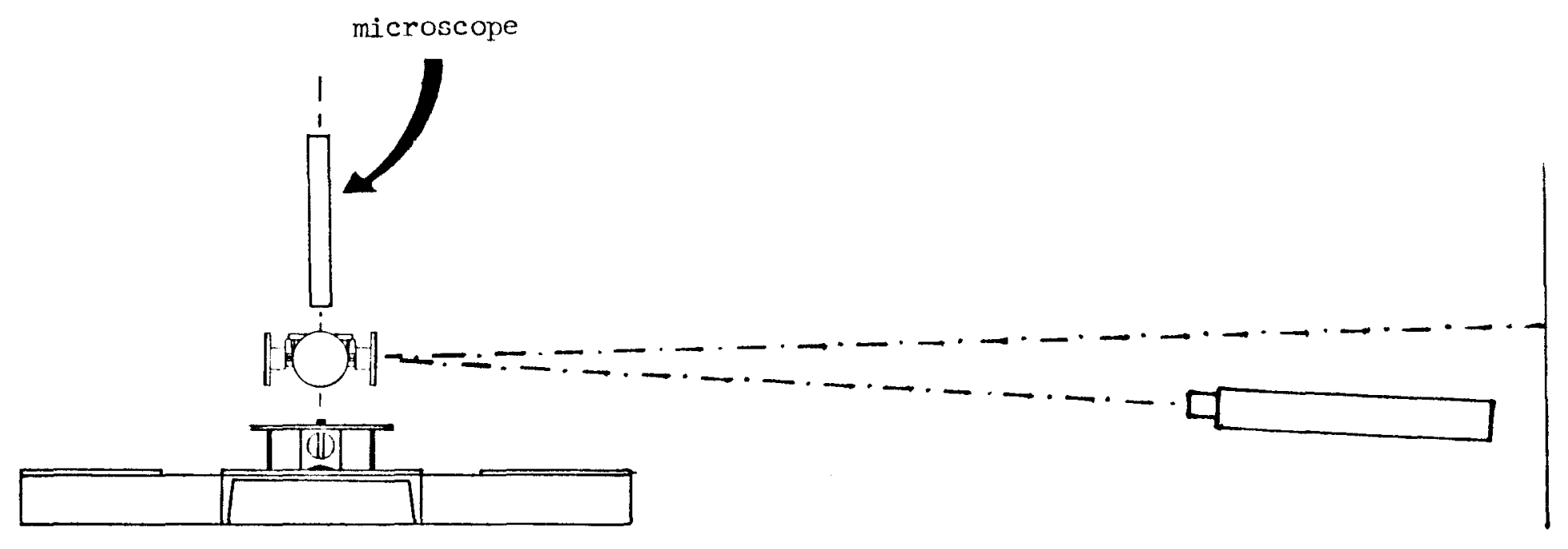

Figure 14:

Diagrammatic View of Apparatus from Anterior Aspect 
the slide projector. The projectors had to be tilted in one plane to avoid positioning of the projected image back onto the body of the device, itself. This arrangement permitted the measurement of transverse axis yaw with either projection system. The transverse axis roll was detected by the anterior projection system while transverse axis pitch was detected by the lateral projection system. Determination of the quantitative values of these parameters was possible by means of the following trignometric calculations (refer to Figures $15 \& 16):$

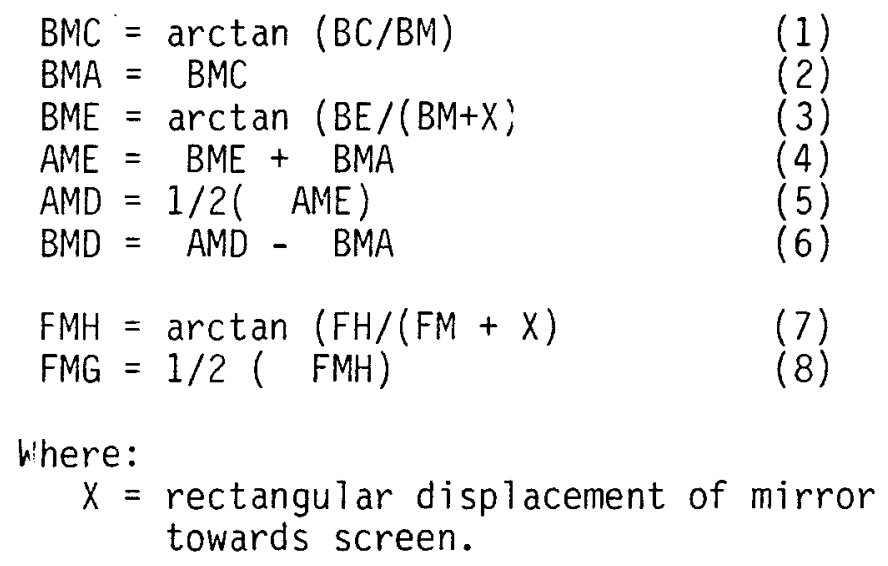

Where:

$X=$ rectangular displacement of mirror towards screen. 


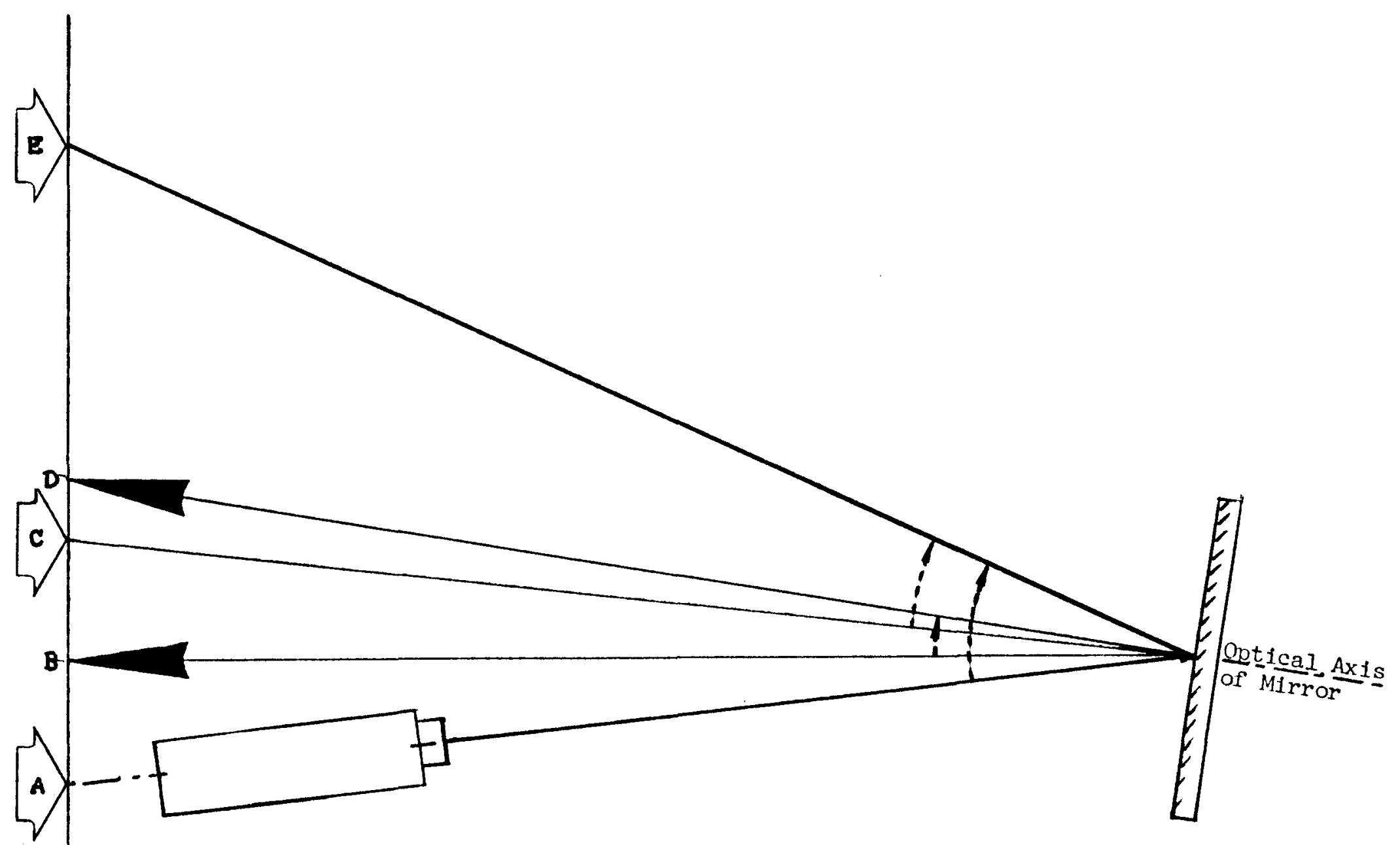

Figure 15: Diagrammatic Depiction of Optical Path (Lateral View)

8 


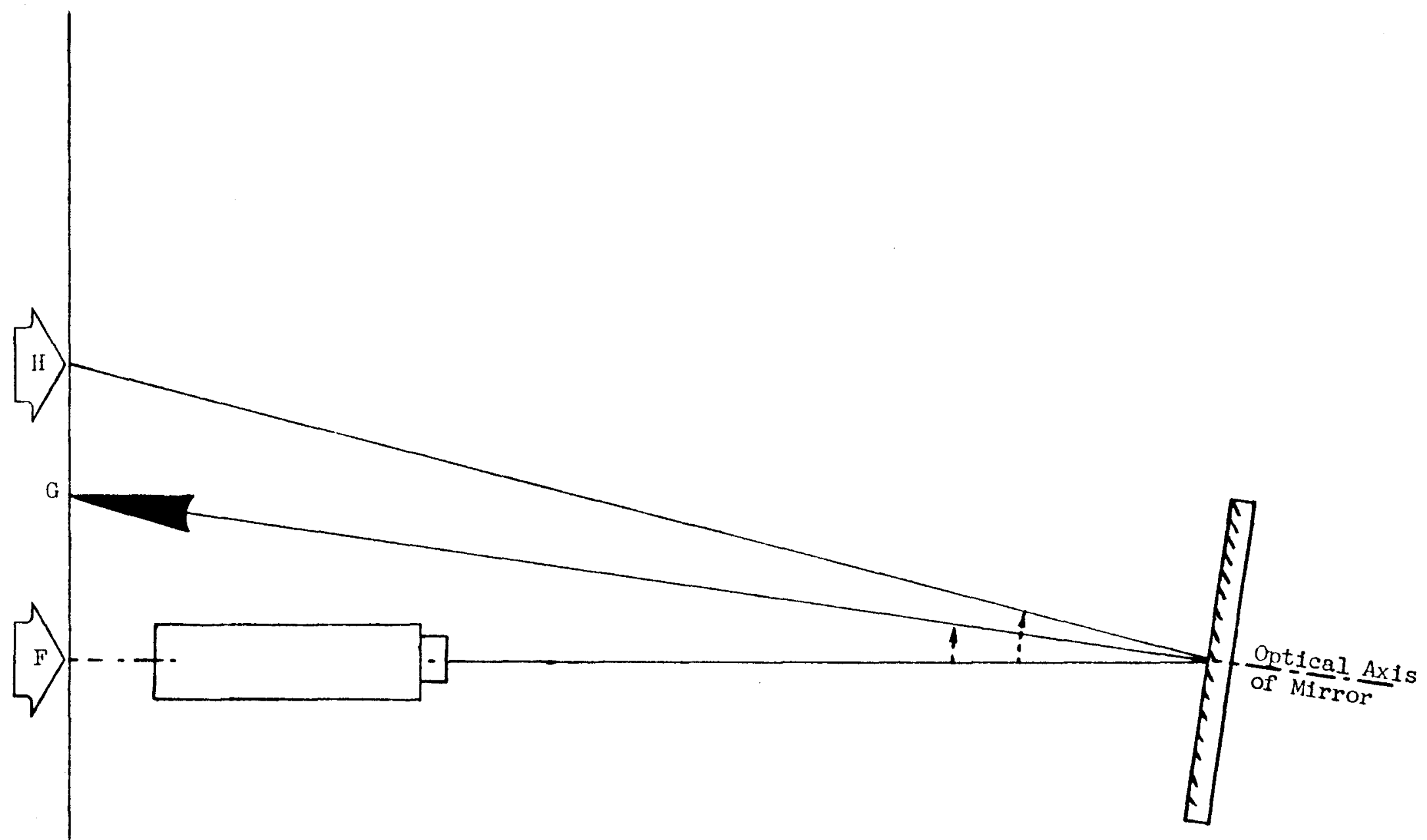

Figure 16: Diagrammatic Depiction of Optical Path (Superior View) 
MATERIALS AND METHODS

\author{
PART 2: \\ Experimental Procedures
}

The effect of fabric screens on the accuracy and dimensional stability of polyether interocclusal records was investigated. The casts of one subject were chosen for this experiment. The rationale for this decision is presented in the discussion chapter of this writing.

Polyether maxillary and mandibular impressions were taken of a 27 year old male. The individual presented with a class I occlusal relationship, minimal restorations, no symptoms of TMJ related pathology, and a locatable kinematic axis. A hinge axis locator was used to determine the location of the kinematic axis. A facebow transfer was then performed using the located axis. Centric relation records were performed at three levels of anterior opening using a Lucia jig and acylic (Dura-lay*) records. The consistency of these records was confirmed by utilizing the split-cast technique (Schwarz, 1975). After the casts were mounted, $110 \mathrm{~mm} \times 25.4 \mathrm{~mm}$ nut was imbedded into the lingual land area of the mandibular cast. A $24 \mathrm{~mm}$ silver/ copper disk** was machined flat and epoxied to the palatal area of the

* Reliance Dental Manuf. Co., Worth, IL

** United States Gov't. Mint, Philadelphia, PA 
maxillary cast. A machine screw was screwed into the nut. The head of the screw engaged the metal disk and acted as a limitation to closure. This limiting assembly was set to an arbitrary height that allowed for non-contact of the occlusal surfaces of the teeth. Cement was then applied to the nut-screwthread interface to prevent variation during experimental manipulation.

Multiple occlusal registrations of the articulated casts were performed. Every odd-numbered registration utilized the polyether registration material*, alone. Each even-numbered registration utilized a commercial fabric screent* in conjunction with the polyether registration material. All procedures involved hinge axis closure of the articulator. The degree of closure was rendered consistent by the limiting screw assembly. In each case, the manufacturer's recommendations were followed exactly (Premier, 1980). The catylist and base were proportioned according to mass. Each record was placed in a $100 \%$ humidity, 27DEG C oven for the first 5 minutes after placement to sumulate oral conditions. Then, each record was stored 30 minutes before being subjected to experimental manipulation. All storage was performed in a room temperature environment.

The maxillary assembly of the modified articulator was weighted so as to balance its vertical force. This resulted in a vertical cast-seating force of newtons. Before the first experimental measurement procedures, the system was initialized by means of a solid plaster jig. The jig was fabricated by plastering together the mounting plates

\footnotetext{
* Premier Dental Products Company, Morristown, PA
}

** Coe Laboratories, Inc., Chicago, IL 
of Whip-mix articulator number 12857 as the articulator's maxillary and mandibular members were related by the two condylar areas and the indisal pin. This represents the undisplaced condition. The jig was then transferred to the measuring system. The microscopes were centered on ICP and the coordinates of the local-global origin coincidence were recorded. The location of the projected cross-hairs was noted on the anterior and lateral screens.

Each record was placed in the device 30 minutes, 6 hours, 24 hours, and 48 hours after its initial mix time. With each proceeding, the $X, Y$, and $Z$ positions of ICP were recorded by means of the microscope assemblies. The positions of the cross-hair center on the anterior and lateral screens were recorded directly on graph paper. These raw data for angular displacement were then acquired by a Microsoft Basic* program driving a high resolution digitizer** (200 points to $25.4 \mathrm{~mm})$. The data were then refined into the familiar pitch, yaw, and roll as per calculations illustrated in figure 15 and appendix.

* Interpreter by Microsoft Associates, Bellevue, WA

** Talos Systems, Inc., Scottsdale, AR 


\section{EXPERIMENTAL RESULTS}

The values for pertinent constants such as distances between mirrors and mass of certain components were determined. The baseline values of ICP location and angular attitude were determined using the plaster calibration jig. In order to ascertain the accuracy of the apparatus, the initialization procedure was repeated 50 times utilizing two experimenters; one who manipulated the microscope and rack and pinion assemblies, and one who read the dial guages and marked the graph paper. In order to achieve a degree of blindness in this determination, the individual manipulating the microscopes was not permitted to view the dial guages. The calculations resulted in an apparent consistency (precision) of approximately $.002 \mathrm{~mm}$ for rectangular measurement and .1 DEG for angular determinations.

The experimental raw data consisted of absolute values for ICP position and pencil marks on graph paper. These values were, respectively, logged and acquired by the computer. The true values for ICP coordinates (in reference to ICP position as determined by plaster-jig initialization) along with $x z$ skew, $x y$ skew, and IC axis rotation were determined by the software. Statistical analysis was then performed on these data. The mean and standard deviation for each batch of samples at each time were determined and presented in Table I. Unpaired t-tests were applied to determine if any significant differences 


\section{TABLE I}

\section{Means and Standard Deviations of the Six Parameters \\ Mandibular Displacement}

\begin{tabular}{|c|c|c|c|c|c|c|}
\hline TIME & $X(\mathrm{~mm})$ & $Y(\mathrm{~mm})$ & $z(\mathrm{~mm})$ & $\operatorname{PITCH}(0)$ & YAW (0) & ROLL (O \\
\hline $\begin{array}{l}1 \mathrm{hr} \\
\text { scrn=0 }\end{array}$ & $\begin{array}{r}-1.65 \\
(.04)\end{array}$ & $\begin{array}{r}-1.35 \\
(.01)\end{array}$ & $\begin{array}{c}-0.82 \\
(.03)\end{array}$ & $\begin{array}{r}-0.50 \\
(.29)\end{array}$ & $\begin{array}{l}0.84 \\
(.13)\end{array}$ & $\begin{array}{l}3.34 \\
(.44)\end{array}$ \\
\hline $\begin{array}{l}1 \mathrm{hr} \\
\text { scrn=1 }\end{array}$ & $\begin{array}{r}-1.65 \\
(.01)\end{array}$ & $\begin{array}{c}-1.35 \\
(.02)\end{array}$ & $\begin{array}{c}-0.82 \\
(.03)\end{array}$ & $\begin{array}{c}-0.37 \\
(.19)\end{array}$ & $\begin{array}{l}0.64 \\
(.58)\end{array}$ & $\begin{array}{l}3.30 \\
(.62)\end{array}$ \\
\hline $\begin{array}{l}6 \mathrm{hr} \\
\mathrm{scrn}=0\end{array}$ & $\begin{array}{r}-1.53 \\
(.89)\end{array}$ & $\begin{array}{c}-1.14 \\
(.02)\end{array}$ & $\begin{array}{c}-1.02 \\
(.06)\end{array}$ & $\begin{array}{c}-0.43 \\
(.48)\end{array}$ & $\begin{array}{l}0.62 \\
(.50)\end{array}$ & $\begin{array}{l}3.63 \\
(.36)\end{array}$ \\
\hline $\begin{array}{l}6 \mathrm{hr} \\
\mathrm{scrn=1}\end{array}$ & $\begin{array}{r}-1.80 \\
(.01)\end{array}$ & $\begin{array}{c}-1.14 \\
(.02)\end{array}$ & $\begin{array}{c}-0.96 \\
(.06)\end{array}$ & $\begin{array}{c}-0.24 \\
(.50)\end{array}$ & $\begin{array}{l}0.61 \\
(.63)\end{array}$ & $\begin{array}{l}3.50 \\
(.60)\end{array}$ \\
\hline $\begin{array}{l}24 \mathrm{hr} \\
\text { scrn=0 }\end{array}$ & $\begin{array}{r}-1.82 \\
(.06)\end{array}$ & $\begin{array}{r}-1.07 \\
(.14)\end{array}$ & $\begin{array}{c}-0.96 \\
(.16)\end{array}$ & $\begin{array}{c}-0.32 \\
(.23)\end{array}$ & $\begin{array}{l}0.88 \\
(.64)\end{array}$ & $\begin{array}{l}3.65 \\
(.44)\end{array}$ \\
\hline $\begin{array}{l}24 \mathrm{hr} \\
\text { scrn=1 }\end{array}$ & $\begin{array}{c}-1.80 \\
(.02)\end{array}$ & $\begin{array}{c}-1.14 \\
(.01)\end{array}$ & $\begin{array}{c}-0.96 \\
(.03)\end{array}$ & $\begin{array}{c}-0.26 \\
(.33)\end{array}$ & $\begin{array}{l}9.61 \\
(.61)\end{array}$ & $\begin{array}{l}3.60 \\
(.36)\end{array}$ \\
\hline $\begin{array}{l}48 \mathrm{hr} \\
\mathrm{scr} n=0\end{array}$ & $\begin{array}{r}-1.81 \\
(.03)\end{array}$ & $\begin{array}{c}-1.61 \\
(.03)\end{array}$ & $\begin{array}{c}-1.02 \\
(.07)\end{array}$ & $\begin{array}{c}-0.49 \\
(.41)\end{array}$ & $\begin{array}{l}0.94 \\
(.44)\end{array}$ & $\begin{array}{l}3.42 \\
(.80)\end{array}$ \\
\hline $\begin{array}{l}48 \mathrm{hr} \\
\text { scrn=1 }\end{array}$ & $\begin{array}{r}-1.77 \\
(.03)\end{array}$ & $\begin{array}{l}-116 \\
(.03)\end{array}$ & $\begin{array}{c}-0.97 \\
(.04)\end{array}$ & $\begin{array}{c}-0.88 \\
(.71)\end{array}$ & $\begin{array}{l}0.92 \\
(.46)\end{array}$ & $\begin{array}{l}3.40 \\
(.57)\end{array}$ \\
\hline \multicolumn{7}{|c|}{$\begin{array}{l}\text { scrn } \\
\text { scrn } \\
\text { mean } \\
\text { dev. } \\
\text { ues in } \\
\text { illary }\end{array}$} \\
\hline
\end{tabular}


existed between the screen and no-screen groups. A significant difference was observed in only two instances: (1) z displacement at $24 \mathrm{hr}$ and (2) $x$ displacement at $48 \mathrm{hr}$.

The data were also subjected to paired t-test determinations The resulting significant comparisons are summarized in Table II. Note that "Experimental Runs 1, 2, 3, and 4, refer to $1 \mathrm{hr}, 6 \mathrm{hr}, 24 \mathrm{hr}$, and $48 \mathrm{hr}$, respectively.

The final refinement involved in the reduction of the data for each displacement into two vector quantities that fully described each positional alteration: the position vector for ICP and the unit vector of the transverse axis (the meaning and application of these quantities will be discussed in Chapter $V$ ). Coefficients for the mean position vectors of each displacement are presented in Table I ( $x, y$, and $z$ ). The mean magnitudes of ICP positional vectors are presented in Table III. The mean coefficients (direction cosines) for the unit vectors of each position of the transverse axis are presented in Table III. Finally, note that Table I presents the full description of each displacement. This format is excellent for reader visualization of each movement. Additionally, the full displacement of the mandible in relation to the maxilla may be described by vector equations. Such a method is most conducive to modelling, simulation, and extrapolation. The necessary parameters for such manipulation are:

1. Positional Vector of ICP

a. Magnitude (resultant) of ICP displacement

b. $\quad V=x i+y j+z k$ 
TABLE II

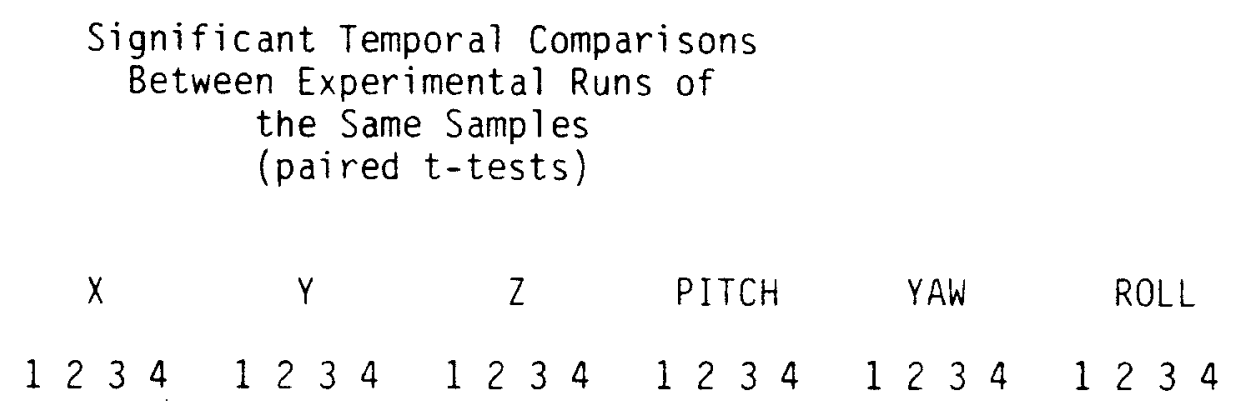

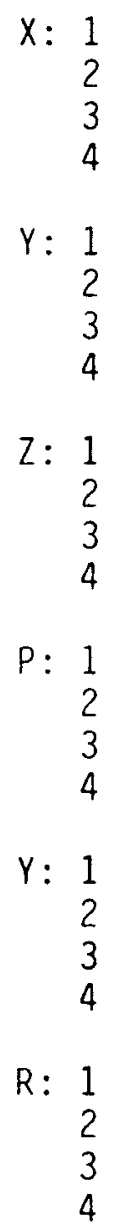

$\otimes \otimes$

$\otimes$

$0 \otimes$

0 


\section{TABLE II I}

Means and Standard Deviations of the Vector Analysis

Parameters of

Mandibular Displacement

\begin{tabular}{|c|c|c|c|c|}
\hline TIME & $\begin{array}{l}\text { ICP Disp. } \\
\text { (mm, absolute) }\end{array}$ & $\operatorname{Cos}(a) p h a)$ & $\operatorname{Cos}($ beta $)$ & $\operatorname{Cos}$ (gamma) \\
\hline $\begin{array}{l}1 \mathrm{hr} \\
\text { scrn=0 }\end{array}$ & $\begin{array}{l}2.00 \\
(.022)\end{array}$ & $\begin{array}{l}-0.0147 \\
(2.23 E-03)\end{array}$ & $\begin{array}{l}1.000 \\
(3.55 E-05)\end{array}$ & $\begin{array}{l}8.67 E-03) \\
(4.98 E-03)\end{array}$ \\
\hline $\begin{array}{l}1 \mathrm{hr} \\
\text { scrn=1 }\end{array}$ & $\begin{array}{l}1.99 \\
(.023)\end{array}$ & $\begin{array}{l}-0.0113 \\
(.0102)\end{array}$ & $\begin{array}{l}1.000 \\
(8.827 E-05)\end{array}$ & $\begin{array}{l}6.43 E-03 \\
(3.36 E-03)\end{array}$ \\
\hline $\begin{array}{l}6 \mathrm{hr} \\
\text { scrn=0 }\end{array}$ & $\begin{array}{l}1.76 \\
(.412)\end{array}$ & $\begin{array}{l}-0.0108 \\
(.794 E-03)\end{array}$ & $\begin{array}{l}1.000 \\
(8.078 E-05)\end{array}$ & $\begin{array}{l}7.468 \mathrm{E}-03 \\
(8.46 \mathrm{E}-03)\end{array}$ \\
\hline $\begin{array}{l}6 \mathrm{hr} \\
\text { scrn=1 }\end{array}$ & $\begin{array}{l}1.904 \\
(.042)\end{array}$ & $\begin{array}{l}-0.0107 \\
(.0111)\end{array}$ & $\begin{array}{l}1.000 \\
(8.772 E-05)\end{array}$ & $\begin{array}{l}4.14 \mathrm{E}-03 \\
(8.65 \mathrm{E}-03)\end{array}$ \\
\hline $\begin{array}{l}24 \mathrm{hr} \\
\text { scrn=) }\end{array}$ & $\begin{array}{l}1.89 \\
(.063)\end{array}$ & $\begin{array}{l}-0.0154 \\
(.0112)\end{array}$ & $\begin{array}{l}1.000 \\
(1.011 E-04)\end{array}$ & $\begin{array}{l}5.51 E-03 \\
(4.02 E-03)\end{array}$ \\
\hline $\begin{array}{l}24 \mathrm{hr} \\
\text { scrn=1 }\end{array}$ & $\begin{array}{l}1.918 \\
(.026)\end{array}$ & $\begin{array}{l}-0.0107 \\
(.0107)\end{array}$ & $\begin{array}{l}1.000 \\
(1.15 E-04)\end{array}$ & $\begin{array}{l}4.55 E-03 \\
(5.69 E-03)\end{array}$ \\
\hline $\begin{array}{l}48 \mathrm{hr} \\
\text { scrn=0 }\end{array}$ & $\begin{array}{l}1.880 \\
(.031)\end{array}$ & $\begin{array}{l}-0.0165 \\
(7.72 E-03)\end{array}$ & $\begin{array}{l}1.000 \\
(1.012 E-04)\end{array}$ & $\begin{array}{l}8.58 E-03 \\
(7.22 E-03)\end{array}$ \\
\hline $\begin{array}{l}48 \mathrm{hr} \\
\text { scrn=1 }\end{array}$ & $\begin{array}{l}1.880 \\
(.039)\end{array}$ & $\begin{array}{l}-0.0161 \\
(8.03 E-03)\end{array}$ & $\begin{array}{l}1.000 \\
(1.461 E-04)\end{array}$ & $\begin{array}{l}3.17 E-03 \\
(0.012)\end{array}$ \\
\hline & \multicolumn{4}{|c|}{$\begin{array}{l}\text { no screen in place } \\
\text { fabric screen utilized } \\
\text { ers not in parentheses } \\
\text { ers in parentheses }\end{array}$} \\
\hline
\end{tabular}


2. Unit Vector of Transverse Axis: $n=\cos ($ alpha $) i+$ $\cos ($ beta $) j+\cos ($ gamma $) k$; where $\cos ($ alpha $)$, $\cos$ (beta), and $\cos$ (gamma) are the direction cosines of $n$ (alpha, beta, and gamma are the angles of $n$ with the $X, Y$, and $Z$ axes, respectively).

3. Rotation of Transverse Axis: "roll"

The mean values of these entities are presented in Table IV. Note that all vector quantities are referenced to the global coordinate system as assigned to the maxillary member. Therefore, all vector quantities pertain to mandibular displacements. 
CHAPTER $V$

DISCUSSION

Instrumentation: Rationale and Design Decisions.

A fill six-degrees-of-freedom measurement of the total displacement between the maxillary and mandibular members of an articulator could have been accomplished by using any of 4 methodologies.

1. Use of a kinematic lingage system with 6 linkages and their associated hardware. The maxillary member would be attached to the first link and the mandibular member to the last link. The raw data would consist of measuring the angular displacements of each linkage. These values, along with the known lengths of the links, would allow for the calculation of displacements of one body relative to the other by means of sequential solving of vector equations (Abler, 1972) or application of transformation matrices (Kinzel, 1972). The most probable implementation of such a system would utilize precision poteniometers or radial optical slot counters. Kinematic linkage systems are best suited to motion (rather than displacement) oriented studies. In light of this emphasis, the investigators who have used such systems did not report the degree of accuracy possible. The degree of accuracy desired when evaluating static inter-occlusal registration materials is more demanding than that of motion studies. Also, the potential expense of the instrumentation involved was prohibitive in the context of their investigation. Therefore, the use of kinematic linkage systems 
in the design of an instrument for measurement of mandibular displacement was rejected.

2. The measurement of the three-dimensional displacement of any 3 points on one member of the articulator relative to the other would allow for a full description of mandibular displacement. This could be accomplished by either of two possible design schemes.

A floating articulator could be prepared similar to the device used in this study. Three points could then be arbitrarily chosen. The points could be located on or off the maxillary member of the articulator; just as long as they bore a consistent relationship to the occlusal arch and transverse axis. The displacement of these 3 points could then be determined by three assemblies similar to the one assembly used in this study (for the determination of ICP displacement). The displacement of these 3 points could then be used to calculate and express positional change of the transverse axis in the format of ICP displacement and pitch, yaw, and roll. Such a set-up would involve the use of 6 measuring microscopes and 9 dial guages. The complexity and cost of such a design led to its rejection from consideration.

Displacement of points attached to the mandibular cast could also be ascertained by application of a mathematical model to a pantograph. The intersections of the pneumatic pointers with the tracing tables of a Denar pantograph actualiy represent points in space that are instantaneousiy common to two reference systems. The global reference system could be assigned to the mandible and its attached screens. The local coordinate system would then consist of the maxilla 
and its pointers. In that the pointer-screen intersection defines the displacement of an axis (that of the pointer) and not a point, the mathematics involved in defining mandibular position would be considerably more complex. Such a difficulty could easily be overcome by the appropriate software. The major experimental technique difficulties encountered in implementing a panto-graph in such an application would involve 2 procedures: (1) Prior to utilization, an extremely exact 3-dimensional mapping of the two bows of the pantograph in relation to their appropriate coordinate system origins would have to be undertaken, and (b) provisions must be made for distortion free magnification and measurement of intercept displacements on the tables.

3. Mandibular displacement could be ascertained by the measurement of the positive and negative intercept displacements of two of the three axes of the articulator with arbitrarily positioned planes positioned perpindicular to the pre-displacement axes. Such a method would involve eight measurements; the two dimensional component vectors of each of 4 line-plane intercepts. A measurement system utilizing such a format was designed, constructed, tested, and subsequently rejected by the investigator in the course of this study. The device consisted of a modified floating Whi-mix articulator on which was mounted 4 projection collimators aligned with the four poles of 2 axes in the horizontal plane (i.e. anterior, posterior, left, and right collimators). Each collimator projected the image of a crosshair onto a screen located approximately $1500 \mathrm{~mm}$ from ICP. Calculations were attempted based on those derived for use on a Buhnergraph (Fattore, 1979). Although the mathematics of such a system proved correct in gross cali- 
bration trials, the device was judged unusable for the following reasons:

a. Since the maxillary member and its collimators measured $2000 \mathrm{~mm}$ by $2000 \mathrm{~mm}$, the device was extremely cumbersome to manipulate.

b. Alignment of the optical axes of the collimators to the appropriate (transverse or anterior-posterior) axes was extremely difficult and possibly not accurate.

c. The beam width of the collimators' crosshairs intersections was, in many instances, wider than its displacement.

d. On several occasions, bumping or jarring of the apparatus altered its alignment. Since the accuracy of the data required a non-varying local coordinate system, any alterations in collimator alignement terminated the usefulness of all data collected prior to a disaligning incident.

This measurement format, for the above-stated shortcomings, rejected.

4. The final method considered in making design decisions involved measurement of the three-dimensional displacement of an arbitrary point in conjunction with the angular displacements of the point's local coordinate system. This would entail the measurement of three linear parameters and three angular parameters. Thus only three dial guages and a simple optical system using projectors or lasers in conjunction with mirrors or beam splitters were necessary. Such a set-up was implemented in this study.

Methods: Rationale of Experimental Procedure.

The experimental portion of this study sought to detect only the effect of fabric screens on the accuracy and stability of polyether inter-occlusal registration material. Clinically, fabric screens are 
usually utilized in centric relation procedures (Hoffman and Reginos, 1972). Therefore, it was considered desirable to simulate CR recording procedures in this experiment. The primary difference between $C R$ interocclusal records and co interocclusal records is that a complete absence of cusp contact is desirable in the former (Fattore, 1979) CR records utilizing a firm dis-occluding stop (Lucia jig) were chosen as a basis for this experiment. It was decided to perform multiple repetitive record procedures on one set of casts mounted in CR on a Whip-mix articulator. The alternative to this format would be to perform record procedures on numerous individuals. This was rejected on the basis of three probable major sources of error and/or inconsistency:

a. It has been shown that the thickness of impression materials has significant effect on dimensional distortions (Ciesco, 1978). Since all interocclusal record materials are derived from impression materials (Balthazar, 1979), one can postulate that the thickness of inter-occlusal records has bearing on the amount of cast positional displacement caused by its distortion. Therefore, if the goal of this study is to properly compare similar record procedures, the thickness of the records must be consistent. This requirement would be extremely difficult to fulfill if CR records were taken of multiple individuals, due to variabilities in clinical $C R$ position and Lucia jig fabrication.

b. The anatomy of the teeth would have effect on the thickness of the material. Different occlusal anatomy schemes may or may not directiy interfere with the fabric matrix. Also, varying embrasure anatomy forms (crowding, diasthemas, etc.) would create varying degrees of "springback" error.

c. Soft tissue variations may or may not create avoidance responses as a reaction to impingement by the framework portions of the fabric screen assemblies.

Since the goal of this study is to assess any effects caused by the fabric screen, itself, the decision was made to perform multiple mani- 
pulations of only one occlusion in a controlled laboratory situation.

Comparison of Methodology and Instrument Design with other Investigations.

Comparison With Motion Studies. The review of the literature revealed that the study of mandibular movement exhibits little standardization in the format of experimental data. Some studies involved depictions of envelopes of motion as drawn by a single point (Posselt, 1952; Knap, 1973; Jankelson, 1979; Salomon, 1979; Jemmt, 1979). Only Jemmt (1979) realized the limitations of point-motion studies in that the principle data derived from his investigation deals with timing parameters of the chewing stroke.

Other investigators constructed mechanisms capable of full measurement of mandibular motion, yet presented their data in a less inclusive format (Abler, 1972). Even though Abler utilized a fully capable method he chose to present his data in the format of envelopes of motion. The clinical applicability of motion envelopes to proper mounting and modeling of the inter-occlusal relationship on an articulator is somewhat limited. Abler's device was, however, used by Beck and Knap (1976) in a clinically relevant study that involved evaluation and comparison of border movements of several patients as related to the border movements of their articulator set-ups. Comparison was made on the basis of motion envelope geometry. Abler (1972) and Messerman (1967) also used electro-mechanical playback devices to demonstrate the comprehensiveness of their measurement systems.

Despite the elaborateness and comprehensive nature of these investigations, their data holds little relevance to clinical situations 
and, more significantly, exhibits no compatability of data for comparison with other studies. The ability of other investigators to design experiments that generate duplicate or comparable data to existing studies is a primary criterion of good scientific investigation. Lee's study (2969) that involved orientation of the recording device to a kinematic axis represented an important step in establishing a clinically relevant format for expreimental data. Since the rotational hinge axis (kinematic axis) is coincident with the articulator transverse axis, Lee's data (McCoy, 1976), like the data in this study, is directly applicable to comparison of the clinical situation to the articulator mounting. This is also demonstrated by the fact that Lee's data were used in establishing the design of a fixed intercondylar semiadjustable articulator (Lundeen, 1979). The Pand-Dent articulator is based on mathematical modeling of the hinge axis by means of guidance applied to fixed condylar analogs. This is based on the concept that the articulator represents a functional (kinematic axis) rather than an anatomical (inter-condylar axis) model of the TMJ articulation. Thus, when a kinematic axis is used, the only function of the articulator is to guide (and thus model) the movements of the articulator transverse axis, not the condyles. This guidance, therefore, need not take place exactly at the analogous locations of the anatomical condyles; it need only be proportional guidance of the transverse axis at an arbitrary inter-"condylar" distance. Another fixed-intercondylar semiadjustable articulator, the Teledyne XP-51 (Tanake, 1979), is based on a concept of proportional, as opposed to anatomical, guidance of the articulator transverse axis. Note that the parameters of 
full description of mandibular motion used in this study (pitch, yaw, roll, $x, y$, and $z$ displacement) are not dependent on any specific location of the condylar elements.

Comparison with Static Studies. The instrument designed and utilized in this investigation examined the end results of a positional alteration of one cast in relation to the other. The static evaluation of mandibular displacement probably has the greatest amount of clinical relevance.

Most qualitative studies involving interocclusal relationships account for the detection of all possible motions of the mandible in that they involve the boolean values of 1 (fit) and 0 (no fit). It is difficult to imagine that the two plaster blocks involved in a splitcast evaluation would fit together perfectly if displacement in one or more of the ICP or transverse axis parameters occurred (Lucia, 1964; Schwarz and Gear, 1975). Thus split-cast technique offers both generation of a consistent (- or 1; yes or no) data format and a clinically relevant (confirmed or not confirmed) procedure. The data derived from this investigation can be adapted to the boolean values of "fit" (no alteration in ICP position or pitch, yaw, and roll), and "no-match" (variation in one or more of the 6 parameters of position). One can conclude, then, that studies involving fit or no-fit data result in data that is, indeed, both clinically relevent and open to experimental duplication.

A much greater variation in data formats is encountered when evaluating quantitative studies involving inter-occlusal relationships. Analysis of two-dimensional tracings and pointer positions such 
as the studies of Kabcenell (1973), and Grasso and Sharry (1968) offer no degree of standardization and no consistent orientation to any anatomic landmark or kinematic axis.

Millstein's experiments $(1971,1973,1975)$ did, indeed, deal with three-dimensional distortions of inter-cast relationships. However, the data was based on displacement of arbitrary cast landmarks. The clinical relationship of these landmarks to an articulator axis, and the resulting clincially relevant distortions, would have been performed on the patient involved. This would have allowed for the refinement of Millstein's data into terms of articulator axis error. A similar comment may be made in regard to the investigations by Strohaver. The volume of measurements taken during each experimental run was more than enough than necessary for calculation of transverse axis distortion. Strohaver chose, however, to present his data by means of irrelevant, arbitrarily defined triangles connecting varying points on the casts and the articulator. Such a format was not conducive to comparison of results with other studies.

Investigations involving the Buhnergraph (and related devices) represent more of an attempt to evaluate inter-cast relationships in terms of transverse axis distortion (Long, 1970; Lundeen, 1974; Remien, 1974; Fattore, 1979). The Buhnergraph is a device that determines the points of intersection of the pre-displacement transverse axis with the post-displacement condylar housing planes (Fig. 17). The application of trignometric analysis to this situation (Hart; in Fattore, 1979) allows for the derivation of refined data that accounts for four of the six parameters of transverse axis displacement used in this 


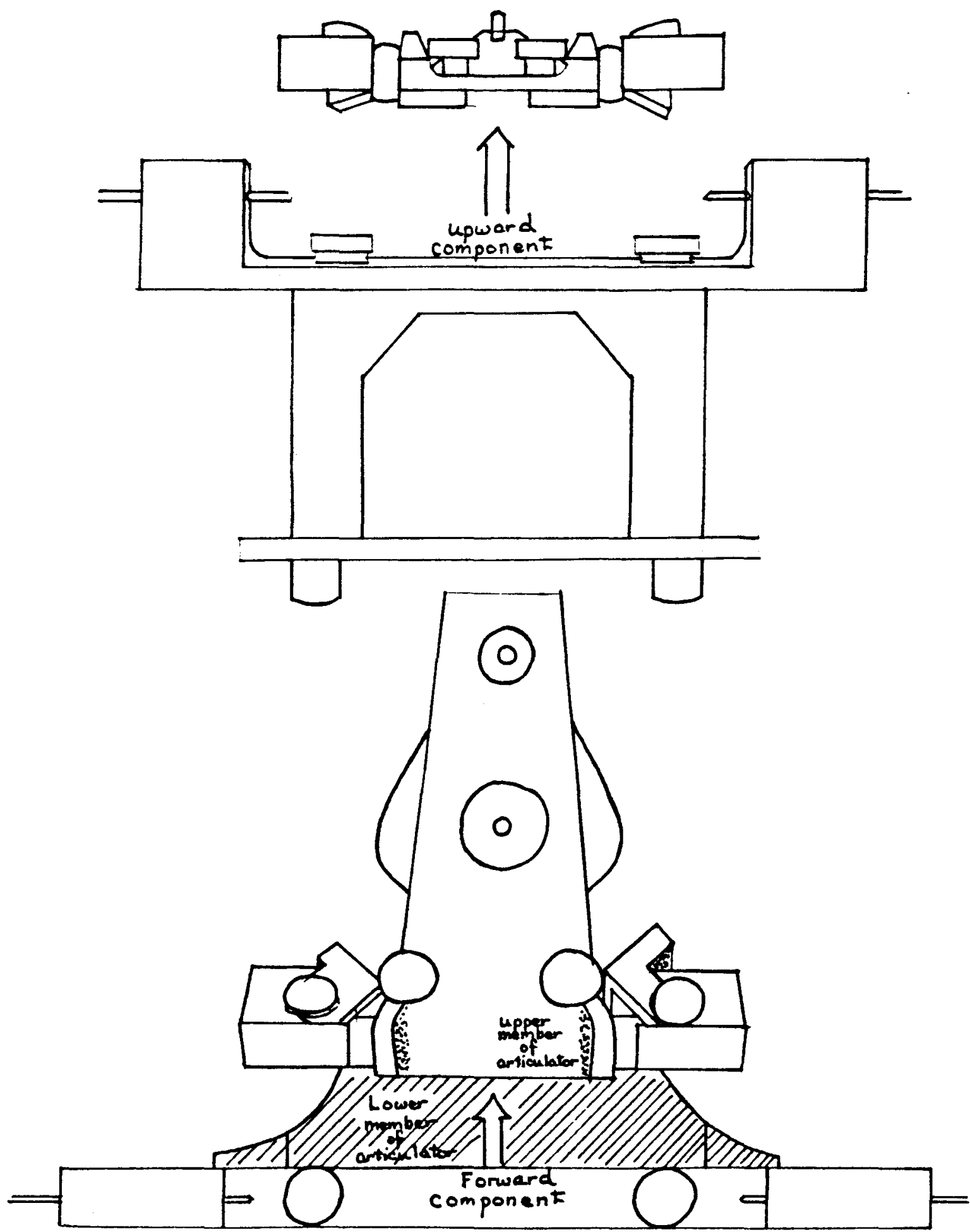

Figure 17: I1lustration of the Buhnergraph 
study as follows:

\begin{tabular}{ll} 
Current Study & Fattore, 1979 \\
\cline { 2 - 2 } X displacement of ICP & x vector \\
$Y$ displacement of ICP & undetectable \\
$Z$ displacement of ICP & Yvector \\
Skew in YZ plane ("pitch") & "Declination" \\
Skew in XY plane ("yaw") & "Rotation" \\
Rotation of transverse axis & undetectable
\end{tabular}

Therefore, it should be noted that an important positive feature of Buhnergraphic studies is that they do evaluate distortions (though incompletely) in terms of transverse axis alteration.

Hoffman's modification of a Ney articulator was also a study that emphasized measurement of transverse axis distortions. In that the full displacements, in three dimensions, of each of the two condylar elements was evaluated, five of the six parameters of axis displacement could be determined from the data. The only displacement that was impossible to detect using Hoffman's apparatus with feeler guages was rotation about the transverse axis.

Thus, it can be stated that the clinical situation involving the mounting of casts on an articulator is open to several types of error. With the exception of articulators used in functional generated path techniques and transographic devices, all articulators have one entity in common: the articulator transverse axis. One could conclude, then, that research devices involved in evaluating interocclusal record materials would most ideally involve evaluation of articulator transverse axis distortions. It was noted that several recent studies have focused the displacement evaluation of the intercast relationship on alterations in position of transverse axis. 
Mathematical Modeling Considerations.

It has been demonstrated that the distortion caused by interocclusal record materials can be described by means of transverse. axis displacement. This is only one method of describing the movement of one member of the articulator relative to the other. ICP displacement and transverse axis skew may not always be the most clinically relevant parameters to derive from an investigation. The experimenter may wish to ascertain, for example, cusp tip displacement or condylar head movement. This is entirely possible using any comprehensive recording system. As long as all aspects of three dimensional displacement were measured, no matter what the method, the investigator can express his results in terms of displacement of any particular points associated with the mandible. Such a goal is easily atainable by the fulfillment of two criteria. The displacement of any point on an object during movement of the object can be determined so long as (1) the rectangular and angular displacement of the object's local reference system is known and (2) the local Cartesian coordinates of the point in question (i.e. mapping of the displacable member) is known (Rogers and Adams, 1976). Manipulation of data in such determinations is accomplished by means of transformation matrices. Kinzel (1972) used transformation matrices to establish the functional anatomy of the dog's articular surface. Since parts of his kinematic linkage were, mechanically, referenced to the members of the articulation, the displacements of given points on the articular surface could be "tracked" by means of transforms applied to the initial coordinates of the articulator surface (as determined by post-experimental dissection and 
three dimensional mapping of the articular surface).

Application of Mathematical Modeling Principles.

Roedema's (1978) computer model of the mandible was accomplished by using an axis-oriented local-global format. The major difference between this study and that of Roedema involves the location of the origin of the local coordinate system; Roedema used the center of the left condyle, while this investigator used ICP. This difference is not very significant since such a choice is really arbitrary. The rationale for choosing ICP dealt with the ease of visualization of the data (It was thought that the reader would better be able to visualize the displacements of the articulator with the use of a bilaterally symetrical arrangement). By deriving an accurate three-dimensional map of a Denar D5A articulator, along with the cusp tips of its mounted casts, Roedema was able to relate movements (i.e. transverse axis displacements) to cusp tip displacements. By application of transformation matrices, the three-dimensional tensor associated with any cusp tip for any movement could be calcualted. A similar manipulation of the refined data was accomplished in the context of this study. By utilizing three-dimensional transformation matrices, along with a rudimentary mapping of the Whip-mix articulator, the experimental displacements that occurred were expressed in terms of left and right (Whip-mix intercondylar distance set at " $\mathrm{M}$ ") condylar displacements and, for the sake of example, the mandibular first molar mesiohuccal cusp tip displacement. In order to accomplish this, three parameters of displacement and attitude in space were necessary.

1. The magnitude and equation of the ICP displacement (position) 
vector. This quantity is simply another format for description of point displacement. The coefficients of the ICP position vector are equal to its displacement in respect to each azis. Therefore the formula is written in the form:

$$
V=X_{i}+Y_{j}+Z k
$$

A direct application of the distance formula yields the magnitude (v) of ICP displacement as follows:

$$
\begin{aligned}
& v=V \\
& v=\operatorname{SQR}(X-0) \star \star 2+(Z-0) \star \star 2
\end{aligned}
$$

2. The unit vector of the transverse axis. The unit vector of a rotational axis is a parameter of its attitude in space. This quantity represents the vector of magnitude 1 that would exist, originating from the origin (global), parallel to the rotational axis. Its direction can be ascertained by the angle it makes with the $X, Y$, and $Z$ axes (alpha, beta, and gamma, respectively). The coefficients of the vector's equation would be, then, $\cos ($ alpha), $\cos ($ beta), and $\cos ($ gamma); since the magnitude is equal to 1 . The formula for the unit vector is:

$$
\begin{aligned}
& N=\cos (\text { alpha }) j+\cos (\text { beta }) j+\cos (\text { gamma }) k \\
& n=N
\end{aligned}
$$

The experimental apparatus measured all non-axial-rotational values (pitch and yaw) of transverse axis angular displacement in respect to the $Y$ axis only. Therefore, the values of the direction cosines, as related to the experimental data, were as follows: 


$$
\begin{aligned}
& \cos (\text { alpha })=\cos (\text { pitch }) \sin (\text { yaw }) \\
& \cos (\text { beta })=\cos (\text { pitch }) \cos (\text { yaw }) \\
& \cos (\text { gamma })=\sin (\text { pitch })
\end{aligned}
$$

The resulting equation for transverse axis unit vector then becomes:

$$
\begin{aligned}
N= & i(\cos (\text { pitch }) \sin (y a w))+j(\cos (\text { pitch }) \cos (y a w)) \\
& +K(\sin (\text { pitch }))
\end{aligned}
$$

2 The rotation of the transverse axis. This value was determined directly from the experimental data (roll) without modification. These formulae were derived for each experimental run, and are presented in appendix A-II.

With the necessary parameters thus determined, it was possible to ascertain the displacement of any mapped point on the mandible by using a generalized rotational transformation matrix $\mathrm{R}$ multiplied by a translation matrix as follows:

$$
\begin{array}{lllllll}
X Y Z H=x y z & 1 & 0 & 0 & 0 & \\
& 0 & 1 & 0 & 0 \\
& 0 & 0 & 1 & 0 & \\
& 1 & m & n & 1
\end{array} \quad F
$$

Where $1, m, n$ are rectangluar $x, y$, and $z$ values for displacement from ICP; and $R$ is the general rotational matrix as presented below.

Only one translation transform was necessary since the rectangular displacement was measured independent of the angular displacement. The general rotation matrix $R$ is derived from multiplication of single rotational transforms as applied to the transverse axis unit vector (this is why it was necessary to derive the formula for the transverse axis unit vector). The elements of the general rotation matrix (as 
it was applied to the transverse axis) are as presented in Table IV. The derivation of this transfer and its modification for use in this investigation will not be presented in that it is beyond the scope. of the context of this paper.

The rectangular coordinates of the left condyle, right condyle, and left first molar mesio-buccal cusp were transformed for each experimental determination. These determinations demonstrate that the displacement of any mapped point on the mandible may be described by means of (1) full measurement of displacement of the local coordinate system (ICP position vector, pitch, yaw, and roll) and (2) use of transformation matrices, derived from these measured displacements, and applied to such mapped points.

Potential_Applications.

This rudimentary example of point displacement description represents significant clinical and research possibilities. The ability to extrapolate the displacement of any point on the mandible during an overall mandibular movement permits the investigator to closely examine local ramifications of generalized motions. Furthermore, the additional ability to standardize and visualize overall mandibular displacement in terms of $x, y, z$, pitch, yaw, and roll arms the investigator with two interchangeable formats for positional analysis. Examples of situations in which these abilities could be used advantageously may include any of the following:

1. Determination of the functional (not anatomical) anatomy of the joint surfaces, as in Kinzel (1972). In his study, Kinzel was able to establish the functional surface contours of the shoulder 


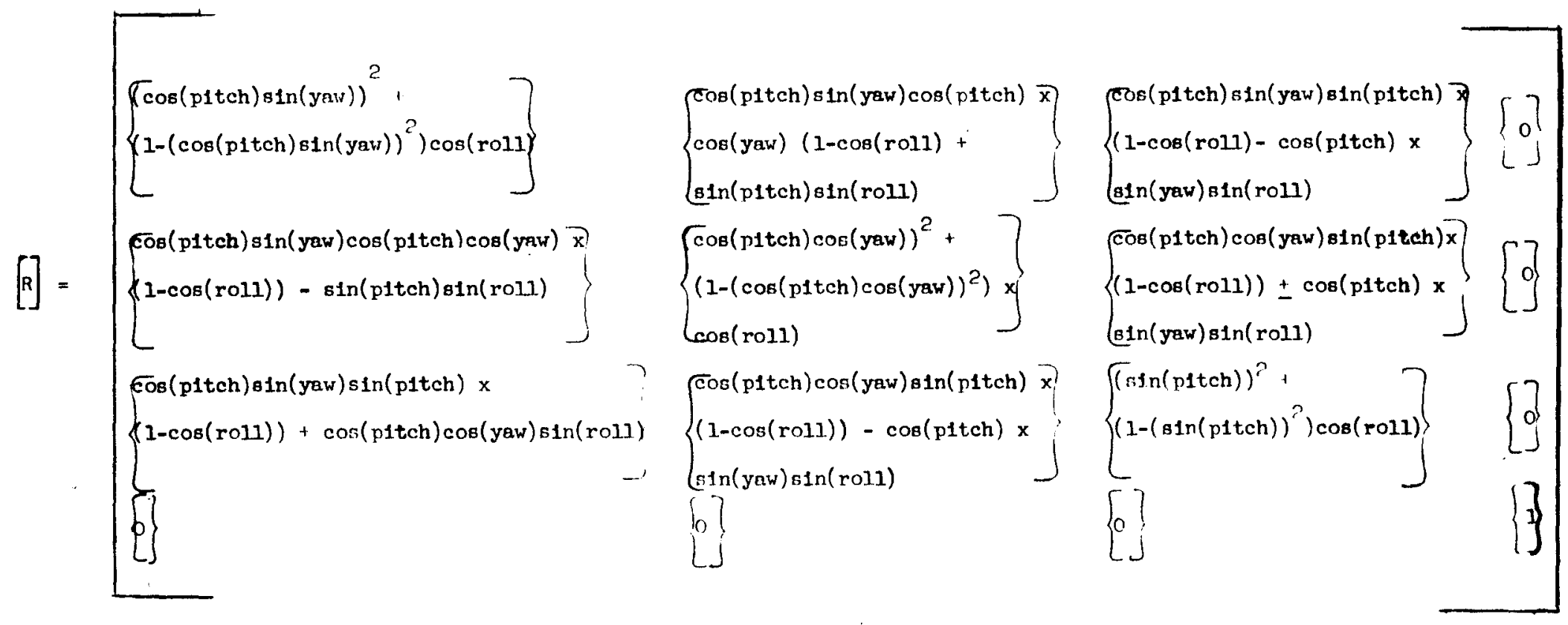

Table IV: The Generalized Rotaticn Transform as Adarted for Specific Use in This Investigation 
joint in dogs. Note that an articulator's condylar areas are really attempted duplicates of functional joint surface contours.

2. Investigations involving the variability of hinge axis locations and CR records could benefit from a comprehensive measurement format for the description of transverse axis displacement. The nature of error in such procedures would be more ascertainable when described in terms of pitch, yaw, rol1, and ICP displacement. Also, results of such studies would exhibit a much higher degree of comparability with the studies of other investigators.

3. The relationships among dental morphology, TMJ anatomy, and mandibular displacement could be investigated with greater clarity. The surface geometry of an interfering incline could, for example, be related directly to condylar displacement or the so-called condylar avoidance path (Guichet, 1979).

4. Motion studies could be rendered more comprehensive by utilizing a fully dezcriptive format of displacement. Abler (1972) extrapolated envelopes of motion from three dimensional data. The converse is also possible, as long as a temporal reference is included in the envelopes' pathes of motion. Since envelopes of motion are really planar intercepts of various mandibular-oriented axes, the actual mandibular motion could be dervied from such traces. Note that such analysis would necessitate consideration of the envelopes created by at least three points as viewed from two directions.

In short, any study that involves mandibular position could benefit from application of a visualizable six degree of freedom descriptive format. Such application would serve several major general purposes: 
a. It would force investigators to design their experiments

to include all aspects of mandibular position.

b. It would greatly enhance comparability of data among different investigations.

c. The determination of mandibular displacement as used in this study allows one to examine the movement of any mapped point on the mandible. Such a modeling and extrapolation ability could be extremely helpful to investigators who wish to examine local ramifications (e.g. cusp tip movement, condylar displacement, etc.) of experimental procedures.

\section{Discussion of Experimental Results.}

The data indicated that the use of fabric screens in polyether interocclusal record procedures caused little or no significant differences $(p=.05)$ in the several types of parameters of mandibular displacement. This is in agreement with Fattore's (2979) data. The possible effects of fabric screens were evaluated by non-paired t-tests comparing each group of screen recrods with each group of no-screen records. No significant difference was noted in any of the angular parameters evaluated: yz skew (pitch), sy skew (yaw), IC axis rotation (roll), and the direction cosines of the IC axis ( $\cos ($ alpha), $\cos ($ beta), and $\cos ($ gamma)). There was no significant differences noted in either the absolute displacement of ICP or the $x$ (left-right) displacement of ICP. Two instances of significance between the screen and no-screen groups were noted: 6 hour z (superior-inferior) displacement and 48 hour y (anterior-posterior) displacement.

When the data were evaluated for significant differences in terms of storage time (paired t-tests), no significance was noted for any of the groups in terms of angular parameters. There did exist, however, significant differences between the first reading and all 
subsequent readings for the $y$ and $z$ displacements in both the screen and no-screen groups. These findings are consistant with those of Balthazar (1979). Anterior-posterior displacements ( $x$ ) exhibited significant differences between the 6 hour reading and all other readings in the no-screen group. The screened records showed significance between the one hour group and all subsequent readings, in addition to a significant difference between the 24 hour reading and the 48 hour reading.

Therefore the following conclusions can be derived from these data:

1. The existence of a fabric screen has no effect on the consistency of polyether interocclusal record materials (in the first 48 hours) in terms of al1 parameters of mandibular displacement.

2. Records using screens exhibited approximately the same patterns of temporal significant differences as records not using fabric screens.

Criticisms and Proposals for Future Investigations.

Experimental Method.

The major purpose of this study was to devise a comprehensive and meaningful format for measurement of mandibular displacement. Additionally, the development and utilization of an apparatus that would conform to such a format was undertaken. The experimental trial, itself, is open to a major criticism involving sample size (especially in respect to the paired t-test $d f$ of 7 ). The number of mixes of polyether should have been four-fold of what was used. This would have allowed for a degree-of-freedom of 31 in each of the paired t-test temporal determinations. 
Instrument Design.

The design of the first instrument served as a significant influence in the design of the second. In consistent calibration results, arduous manipulation procedures, and the investigator's intuition lead to the following criteria regarding the design of the second instrument for measuring the full displacement of one cast in relation to the other:

1. The active area of the device must not be bulky, rather its manipulation should be as similar as possible to that of the articulator that it is evaluating.

2. The realization of $x, y$, and $z$ ICP displacement should be accomplished without physically contacting the articulator portion of the apparatus.

3. Aluminum is not a suitable material in that it is too easily deformed during mishaps.

4. The apparatus should allow for easy adjustments, alterations, and design modifications.

All these criticisms were taken into account in designing and building the second instrument. There exists, however, several minor criticisms of the instrument as it was used in this investigation.

All elements of the optical system should have been related to the main base assembly. Failure to do this allowed for potential error caused by distortion in the floor. Additionally, it was impossible to be absolutely sure that either or both slide projectors were not jarred between experimental runs (although, they were each anchored on 90 lbs. of cement bricks). All the optical measurements of this experiment were made under the assumption that the slide projector reticles were lined up exactly on the optical axis of each lens system. This condition could not be absolutely assured in both the commercial 
slide projectors used.

It also would have been advantageous to use a single light source in conjunction with a beam splitting prism. This would negate the effects of differences in the slide projectors and their internal alignment.

Finally, a narrower representation of the central beam of the light source(s) would have rendered precise location of the beam displacements more consistent.

The existence of these problems and their potential solution is what led to the "Swiss-cross" design of the mounting table. This design, along with the machined steel plates, permitted the versitility of enacting future anticipated changes in the apparatus' set-up.

\section{Future Application.}

The use of a Helium-Neon measurement grade laser with a beam width of $.08 \mathrm{~mm}$ is anticipated in a subsequent study (Fig. 18). A laser would solve the previously mentioned problems as follows:

1. Only a single light source will be used.

2. All appropriate optical components will be mounted on the single steel base table.

3. The use of coherent light will eliminate the problems of beam axis alignment. Simple pin-hole collimation will be possible to further narrow the beam.

4. The displacement of both anterior and lateral beams will be acquired simultaneously on the surface of the digitizer.

Finally, the cost factor of utilizing a measurement grade He-Ne laser is minimal. In fact, the cost of one laser is less than the cost of the two slide projectors used in this investigation. 


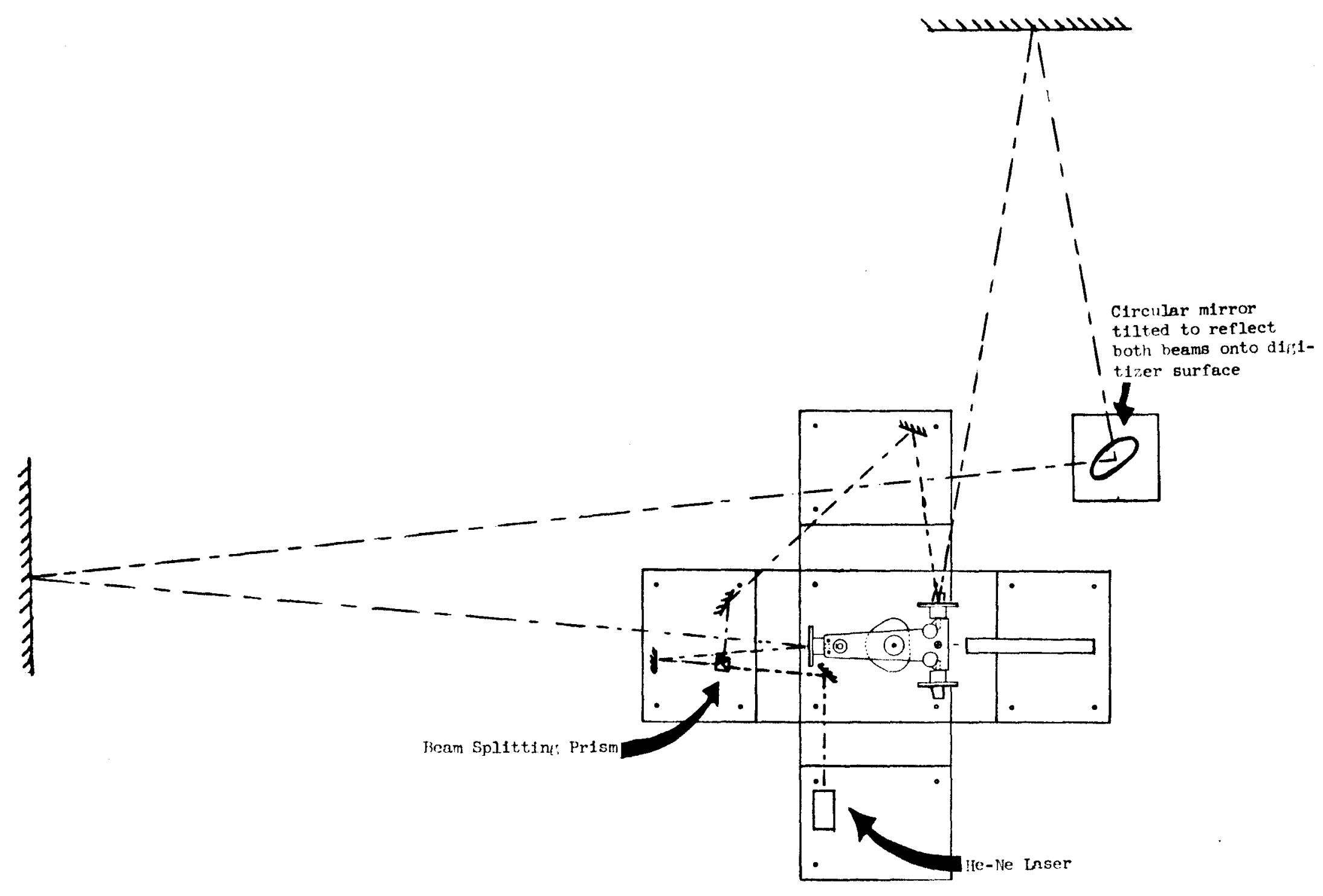

Figure 18: Schematic of Proposed System for More Accurate Angular Measurement 
CHAPTER VI

\section{CONCLUSION}

The following conclusions can be made from this investigation:

1. The review of the dental literature revealed a lack of consistency for describing mandibular displacement.

2. A ful1, six-degrees-of-freedom format for the description of mandibular displacement was formulated. This involved the assignment of two coordinate systems to a floating articulator: the local system was referenced to the mandibular member. Both systems were coincident in the non-displaced condition and shared the intercondylar mid-point (ICP) as their origins. Displacement of the maxillary member relative to the mandibular member could then be described by the noncoincidence of the global and local coordinate systems as follows:

a. $x, y$, and $z$ displacement of the local ICP relative to the global ICP.

b. pitch, yaw, and roll of the $y$-axis (articulator transverse axis).

3. An instrument that measured these parameters was designed, constructed, tested, and utilized.

4. A simple experiment demonstrated that fabric screens do not significantly $(p=.05)$ alter the performance of a polyether interocclusal record material.

5. A second format of displacement analysis, compatible with the first, was derived for the purpose of applying transformation 
matrices to mapped points on the mandibular assembiy in order to ascertain specific local displacements. This system involved vector parameters as follows:

a. $\quad v=V=$ magnitude of ICP displacement

b. $\quad V=x i+y j+z k ;$ (ICP positional vector)

c. $n=\cos ($ alpha $) i+\cos ($ beta $) j+\cos ($ gamma $) k ;$ (transverse axis unit vector)

d. Roll = rotation of transverse axis.

An example was executed using the derived transformation matrices as applied to three chosen points on the mandibular member.

6. Future studies involving improvements in the optical portion of the apparatus is anticipated. 


\section{REFERENCES}

1. Abler, J. H.: "Design and Analysis of a Clinical System to Measure and Reproduce Mandibular Motion." Phd Dissertation, Marquette University School of Engineering, Milwaukee, Wisconsin, 1972 .

2. Andriacchi, T. P., Hampton, S. J., Schultz, A. D., and Galente, J. 0.: "Three Dimensional Coordinate Data Processing in Human Motion Analysis." Journal of Biomechanical Engineering 101:279-83, 1979.

3. Barbenel, J. C.: "The Biomechanics of the Temporomandibular Joint - A Theoretical Study." Journal of Biomechanics $8: 251-58,1972$.

4. Beck, M. 0.: "Clinical Evaluation of the Arcon Concept of Articulation." Journal of Prosthetic Dentistry 9:409, 1959.

5. Berman, M. N.: "Accurate Interocclusal Records." Journal of Prosthetic Dentistry 10:620-30, 1960.

6. Beck, D. B., and Knap, F. J.: "Reliability of Fully Adjustable Articulators Using a Computerized Analysis." Journal of Prosthetic Dentistry 35:630-42, 1976.

7. Bock, V. 0., Ortlieb, R., and Erlangen, K. 0.:"Untersuchungen Uber Die Brauchbarkeit Des Mandibular Kinesiographen (MKG)." Deutches Zahnarztel 33:639-42, 1970.

8. Brewer, A. A.: "Prosthodontic Research in Progress at the School of Aerospace Medicine." Journel of Prosthetic Dentistry 13:50-69, 1963.

9. Brotman, D. N.: "Hinge Axes Part I. The Transverse Hinge Axes." Journal of Prosthetic Dentistry 10:430-40, 1960.

10. Celenza, F. V.: "An Analysis of Articulators." Dental Clinics of North America 23-2:305-28, 1979.

11. Christensen, P. B.: "Accurate Casts and Positional Relation Records." Journal of Prosthetic Dentistry 8:475-82.1958.

12. Cohen, J.: "The Hinge Axis and Its Practical Application in the Determination of Centric Relation." Journal of Prosthetic Dentistry $10: 248-58,1960$. 
13. Craddock, F. W. and Symmons, H. F.: "Evaluation of the Face Bow." Journal of Prosthetic Dentistry 2:634, 1952.

14. Dawson, P. E.: "Centric Relation - Its Effect on Occluso Muscle Harmony." Dental Clinics of North America 23-2:169-80, 1979.

15. DePietro, A. J.: "The Articulator as a Dental Instrument not a Dental Philosophy." Dental Clinics of North America 23-2:213-29, 1979.

16. Desouza, P. V. and Houghton, P.: "Computer Location of Medial Axes." Computers and Biomedical Research 10:333-43, 1977.

17. Freudenstein, F. and Woo, L. S.: "Kinematics of the Human Knee Joint." Bullet in of Mathematics and Biophysics 31:215-32, 1969.

18. Gelb, H.: "Occlusal Imbalance and TMJ Dysfunction." Dental Clinics of North America 23-2:181-97, 1970.

19. George, J. P. and Boone, M.: "A Clinical Study of Rest Position Using the Kinesiograph and Myomoniter." Journal of Prosthetic Dentistry $41: 456-72,1979$.

20. Granger, I. R.: "The Principles of Obtaining Occlusion in Occlusal Rehabilitation." Journal of Prosthetic Dentistry 13:714-18, 1963.

21. Grant, P. C.: "Biomechanical Significance of the Instantaneous Center of Rotation - the Human Temporomandibular Joint." Journal of Biomechanics 6:109-13, 1973.

22. Grasso, J. E., and Sharry, J.: "The Duplicability of Arrow-Point Tracings in Dentulous Subjects." Journal of Prosthetic Dentistry 20:106-15, 1968 .

23. Gregory, G., Shryock, E. F., and Daum, L.: "Hinge Axis Analys is New Procedures for Location." Journal of the Southern California Dental Association 37:58-86, 1969.

24. Guichet, N.: "Occlusion." The Denar Corporation, Anaheim California, 1977.

25. Guichet, N.: "The Denar System and its Application in Everyday Dentistry." Dental Clinics of North America 23-2:243-58, 1979.

26. Harcourt, J. K.: "Accuracy in Registration and Transfer of Prosthetic Records." Australian Dental Journal 22:182-90, 1974.

27. Helkimo, M., Ingerval1, B., and Carlsson, 0.: "Comparison of Different Methods in Active and Passive Recording of the Retruded Position of the Mandible." Scandinavian Journal of Dental Research 81: 265-77, 1973. 
28. Hickey, J. C., Allison, H. L., and Woelfel, J. N.: "Manibular Movements in Three Dimensions." Journal of Prosthetic Dentistry 13:72$91,1963$.

29. Hoffman, P. J., Silverman, S. I., and Garfinke1, L.: "Comparison of Condylar Position in Centric Relation and in Centric Occlusion in Dentulous Subjects." Journal of Prosthetic Dentistry 30:582-88, 1973.

30. Hungerford, J. C.: "Graphic Manipulations Using Matrices." Byte 3: $156-67,1978$.

32. Ingerval1, B., Helkimo, M., and Carlsson, G. E.: "Recording of the Retruded Position of the Mandible with Application of Varying External Pressure to the Lower Jaw." Archives of Oral Biology 16: 1165-72, 1971.

33. Isaacson, R. J., Erdman, A. G., and Hultgren, B.: "Kinematics of Jaw Growth." Journal of Biomechanical Engineering 100:93-8, 1978.

34. Jankelson, H.: "Neuromuscular Aspects of Occlusal Effects of Occlusal Position on the Physiology and Dysfunction of the Mandibular Musculature." Dental Clinics of North America 23-1:157-68, 1979.

35. Jemt, T., Karlsson, S., and Hedegard, D.: "Mandibular Movements of Young Adults Recorded by Intraorally Placed Light Emitting Diodes." Journal of Prosthetic Dentistry 42:669-73, 1979.

36. Kabcenel1, J. L.: "Effect of Clinical Procedures on Mandibular Position." Journal of Prosthetic Dentistry 14:266-78, 1964.

37. Kantor, M. E., Silverman, S. I., Garfinkel, L.: "Centric Relation Recording Techniques - A Comparitive Investigation." Journal of Prosthetic Dentistry 28:593-600, 1972.

38. Kass, C. A. and Knap, F. J.: "Analysis of Occlusion Before and After Occlusal Adjustment." Journal of Prosthetic Dentistry 32:16370,1974 .

39. Kinzel, G. L., Hall, A. S., and Hillberry, B. M.: "Measurement of the Total Motion Between Two Body Segments - I - Analytical Development." Journal of Biomechanics 5:93-105, 1972.

40. Kinzel, G. L., Hall, A. S., and Hillberry, B. M.: "Measurement of the Total Motion Between Two Body Segments - II - Description of Application." Journal of Biomechanics $5: 283-93,1972$.

41. Knap, F.: "Study of Mandibular Motion in Six Degrees of Freedom." Journal of Dental Research 49:289-92, 1970.

42. Lauretzen, A. G. and Wolford, L.W.: "Occlusal Relationships - The Split Cast Method for Articulator Techniques." Journal of Prosthetic Dentistry 14:256-65, 1964. 
43. Lee, R. L.: "Jaw Movements Engraved in Solid Plastic for Articulator Controls. Part 1: Recording Apparatus." Journal of Prosthetic Dentistry 22:209-24, 1969.

44. Lemmer, J., Lewin, A., and Vanresburg, L. B.: "The Measurement of Jaw Movement. Part I." Journal of Prosthetic Dentistry 36:211-18, 1976.

45. Lemmer, J., Lewin, A., and Vanresburg, L. B.: "The Measurement of Jaw Movement. Part II." Journal of Prosthetic Dentistry 36:312$18,1976$.

46. Long, J. M.: "Location of the Terminal Hinge Axis by Intraoral Means." Journal of Prosthetic Dentistry 23:11-24, 1970.

47. Lucia, V. 0.: "Centric Relation Theory and Practice." Journal of Prosthetic Dentistry 10:849, 1960.

48. Lucia, V. 0.: "Principles of Articulators." Dental Clinics of North America 23-2:199-212, 1979.

49. Lundeeen, H. C.: "Mandibular Movement Recordings and Articulator Adjustments Simplified." Dental Clinics of North America 23-2: $199-212,1979$.

50. Lundeen, H. C.: "Centric Relation Records: The Effect of Muscle Action." Journal of Prosthetic Dentistry 31:244-53, 1974.

51. Lunquist, D. 0. and Fieberger, G. E.: "Registrations for Relating the Mandibular Cast to the Maxillary Cast Based on Kennedy's Classification System." Journal of Prosthetic Dentistry 35:373-5, 1976.

52. McCollum, B. B. and Stuart, C. E.: "A Research Report." South Pasadena Scientific Press 1955.

53. McCoy, R. B., Shryock, E. F., and Lundeen, H. C.: "A Method of Transferring Mandibular Movement Data to Computer Storage." Journal of Prosthetic Dentistry 36:510-16, 1936.

54. Millstein, P.L., Kronman, J. H., and Clark, R. E.: "Hydropic Measuring System for Testing The Accuracy of Interocclusal Recording Mediums." Journal Dental Research 49:462, 1970.

55. Millstein, P.L., Clark, R. E., and Kronman, J. H.: "Determination of the accuracy of Wax Interocclusal Registrations Part II." Journal of Prosthetic Dentistry 29:40-5, 1973.

56. Millstein, P.L., Kronman, J.H., and Clark, R. E.: "Determination of the accuracy of Wax Interocclusal Registrations Part I." Journal of Prosthetic Dentistry 25:189-96, 1971. 
57. Millstein, P.L., Clark, R. E., and Myerson, R. L.: "Differential Accuracy of Silicone Body Interocclusal Records and Associated Weight Loss Due to Volatiles." Journal of Prosthetic Dentistry 33: $649-54,1975$.

58. Moss, M. L.: "A Functional Cranial Analysis of Centric Relation." Dental Clinics of North America 19:431-42, 1975.

59. Nevakari, K.: "Elapsio Prearticularis of the Temporomandibular Joint." Acta Odont Scandanavia 18:123-70, 1960.

60. Nielsen, K. L. and Vanlonkhuyzen, J. H.: "Plane and Spherical Trigonometry." Barns and Noble Books, New York, New York, 1954.

61. Orin, D. E., McGhee, R. D., Vukobratovic, M., and Hatroch, C.: "Kinematic and Kinetic Analysis of Open Chain Linkages Utilizing Newton-Euler Methods." Matters in Bioscience 43:107-30, 1979.

62. Panjabi, M. M.: "Centers and Angles of Rotation of Body Joints A Study of Errors and Optimization." Journal of Biomechanics 12: 911-20, 1979 .

63. Parker, M. L., Hemhil1, C. D., and Regli, C. P.: "Anteroposterior Position of the Mandible as Related to Centric Relation Registrations." Journal of Prosthetic Dentistry 31:262-5, 1974.

64. Posselt, U.: "An Analyzer for Mandibular Positions." Journal of Prosthetic Dentistry 7:368-76, 1957.

65. Posselt, U.: "Range of Movement of the Mandible." Journal of the American Dental Association 56:10-3, 1958.

66. Posselt, U.: "Studies in the Mibility of the Human Mandible." Acta Odontology 10 (Supplement 11):19, 1952.

67. Preston, J.: "A Reassessment of the Mandibular Transverse Horizontal Axis Theory." Journal of Prosthetic Dentistry 41:805-13, 1979.

68. Remien, J. C. and Ash, M.: "Myo-monitor Centric - An Evaluation." Journal of Prosthetic Dentistry 31:137-45, 1974.

69. Roedema, W. M., Knapp, J. H., Spencer, J., and Dever, M. K.: "Computer Graphic Representation of Mandibular Movements in Three Dimensions Part I.: The Horizontal Plane." Journal of Prosthetic Dentistry $38: 370-83,1978$.

70. Roedema, W. M., Knapp, J. H., Spencer, J., and Dever, M. K.: "Computer Graphic Representation of Mandibular Movements in Three Dimensions Part II.: The Saggittal and Frontal Planes." Journal of Prosthetic Dentistry 40:385-90, 1978. 
71. Solomon, J. A., Waysenson, B. D., and Warshaw, B. D.: "Computer Monitered Radionuclide Tracking of Three Dimensional Mandibular Movements." Journal of Prosthetic Dentistry 41:403-9, 1979.

72. Schwarz, W. D. and Gear, V. D. A.: "Accurate Location and Relocation of Casts on Articulators." Journal of Prosthetic Dentistry 34: $695-97,1975$.

73. Shanahan, E. J. and Leff, A.: "Interocclusal Records." Journal of Prosthetic Dentistry 10:842-8, 1960.

74. Stepanenko, Y. U. and Vukobratovic, M.: "Dynamics of Articulated Open-chain Mechanisms." Mathematics and Biosciences 28:137-70, 1970.

75. Stern, J.: "Editorial Response to Biomechanical Significance of the Instantaneous Center of Rotation by Grant (1973)." Journal of Biomechanics 7:109-10, 1974 .

76. Strohaver, R. A.: "A Comparison of Articulator Mountings Made with Centric Relation and Myocentric Posidito Records." Journal of Prosthet ic Dentistry 28:379-90, 1972.

77. Stuart, C. E.: "Use of the Stuart Articulator in Obtaining Optimal 0cclusion." Dental Clinics of North America 23-2:259-70, 1979.

78. Tanaka, H.: "The Teledyne Articulator System 154 as Applied to Complete Denture Construction." Dental Clinics of North America 23-2:289-303, 1979.

79. Vicker, J. J., Denavit, J., and Hartenberg, R. S.: "An Iterative Method for the Displacement Analysis of Spatial Mechanisms." Journal of Applied Mechanics 63:309-14, 1964.

80. Walker, W.: "Discrepancies Between Arbitrary and True Hinge Axis." Journal of Prosthetic Dentistry 43:279-85, 1980.

81. Waysenson, B. and Solomon, J.: "Three-dimensional Recordings of Envelopes of Motion Related to Mandibular Movements." Journal of Prosthetic Dentistry 38:52-60, 1977. 
102

APPROVAL SHEET

The thesis submitted by Timothy 0 . Hart has been read and approved by:

William F. Malone Ph. D., D.D.S.

James L. Sandrik Ph. D.

Douglas C. Bowman Ph. D.

The final copies have been examined by the director of the the is committee and the signature which appears below verifies the fact that any necessary changes have been incorporated, and that the the is is now given final approval with reference to content, form and mechanical accuracy.

The thesis is therefore accepted in partial fulfillment of the requirements for the Degree of Master of Science.
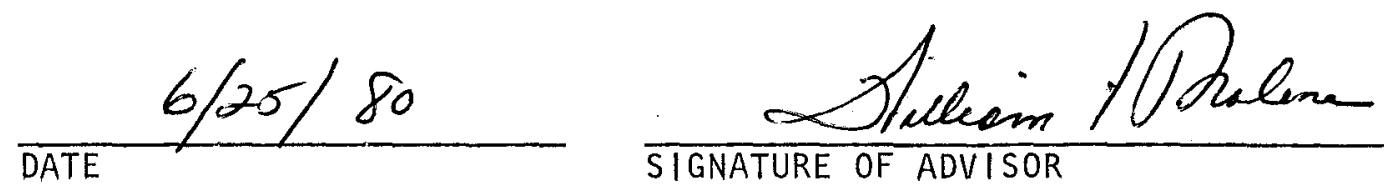

SIGNATURE OF ADVISOR 